\title{
Diagnostic and Prognostic Circulating MicroRNA in Acute Stroke: A Systematic and Bioinformatic Analysis of Current Evidence
}

\author{
Jorin Bejleri, ${ }^{\mathrm{a}, \mathrm{b}}$ Elisabeth Jirström, ${ }^{\mathrm{a}, \mathrm{c}}$ Paul Donovan, ${ }^{\mathrm{a}, \mathrm{c}}$ David J. Williams, ${ }^{\mathrm{b}}$ Shona Pfeiffer ${ }^{\mathrm{a}, \mathrm{c}}$ \\ ${ }^{a}$ Department of Physiology and Medical Physics, Royal College of Surgeons in Ireland, Dublin, Ireland \\ ${ }^{b}$ Department of Geriatric \& Stroke Medicine, Beaumont Hospital, Royal College of Surgeons in Ireland, Dublin, Ireland \\ 'Centre for Systems Medicine, Royal College of Surgeons in Ireland, Dublin, Ireland
}

Background and Purpose Stroke is the second leading cause of death and disability worldwide and its diagnosis, and assessment of prognosis, remains challenging. There is a need for improved diagnostic and prognostic biomarkers. MicroRNAs (miRNAs) play important roles in the post-transcriptional regulation of gene expression and their secretion and remarkable stability in biofluids highlights their potential as sensitive biomarkers in the diagnosis and prognosis of acute stroke.

Methods We carried out a systematic review to assess current evidence supporting the potential of miRNAs to act as unique diagnostic and prognostic biomarkers in blood samples collected from patients suffering acute stroke within 24 hours of symptoms onset.

Results We identified 22 studies eligible for inclusion with 33 dysregulated miRNAs having diagnostic potential in the acute phase of the disease. We identified miR-16, miR-126, and miR-335 as having the highest sensitivity as diagnostic and prognostic biomarkers in acute ischaemic stroke and present original bioinformatic and pathway enrichment analysis of putative miRNA-target interactions.

Conclusions miRNAs represent unique biomarkers which have a promising future in stroke diagnosis and prognosis. However, there is a need for more standardized and consistent methodology for the accurate interpretation and translation of miRNAs as novel specific and sensitive biomarkers into clinical practice.

\author{
Correspondence: Shona Pfeiffer \\ Department of Physiology and \\ Medical Physics, Royal College of \\ Surgeons in Ireland, 123 St. \\ Stephen's Green, Dublin, Ireland \\ Tel: +353-1-402-5125 \\ Fax: +353-1-402-2447 \\ E-mail: shonapfeiffer@rcsi.ie \\ https://orcid.org/0000-0002-8728- \\ 1620
}

Received: December 19, 2020 Revised: February 27, 2021 Accepted: March 19, 2021

Keywords miRNA; Ischaemic stroke; Stroke; Biomarker; Diagnostic; Prognostic

\section{Introduction}

Stroke is the second leading cause of death and disability-adjusted life-years worldwide.' Ischaemic stroke (IS) accounts for $85 \%$ of all strokes, while haemorrhagic stroke (HS) accounts for $10 \%$ to $15 \%{ }^{2}$ Cerebral ischaemia leads to the development of a complex cascade of pathological events centring around neuronal excitotoxicity and adenosine triphosphate depletion. ${ }^{3}$ The ischaemic core is surrounded by the ischaemic penumbra, an area of functionally compromised, partially-perfused salvageable tissue destined for infarction without intervention. Elucidation of the mediators of ischaemic cell death within the penumbra represents a promising area for the development of gene-targeted intervention for neuroprotection. ${ }^{4}$ Furthermore, the damage that occurs during HS is primarily a result of the rapid accumulation of blood and consequent disruption of brain 
tissue and increased local pressure. At present, the diagnosis of stroke relies on the clinician's expertise in patient assessment and rapid access to diagnostic imaging techniques such as computed tomography (CT), CT angiography, CT perfusion or magnetic resonance imaging (MRI). However, clinical assessment of stroke is not always straightforward, since CT images are often normal in early presentation and access to CT perfusion and MRI is still challenging. An accurate, reliable and timely blood biomarker for stroke in the acute setting is currently lacking, although its utility has long been recognised for the purpose of early diagnosis and prognosis, as treatment of acute ischaemic stroke (AIS) is time dependent. ${ }^{5}$

MicroRNAs (miRNAs) are short (approximately 20 to 23 base pair) single-stranded non-coding RNAs that play an important role in the post-transcriptional regulation of gene expression through interaction between the miRNA seed region and complementary sites in target messenger RNA (mRNAs), targeting the mRNA for degradation or translational repression. ${ }^{6}$ The ability of a single miRNA to modulate the expression of multiple genes in multiple pathways places miRNAs in a powerful position in the regulation of all major cellular functions. ${ }^{7}$ The onset of stroke triggers a complex series of physiological, biochemical, and gene expression changes precipitating the onset of neuronal injury, placing miRNAs in an advantageous position in the regulation of multiple neuroprotective and deleterious pathophysiological processes impacting on brain injury maturation. There is a growing body of evidence supporting the potential roles for miRNAs in the attenuation of neurological deficit, modulating key pathways central to the expansion of the ischaemic core. miRNAs dysregulated following ischaemia have been shown to suppress microglial function, playing an important role in the recovery phase by promoting the growth of neuronal tissue, and in the repair of injured brain tissue in the acute phase by stimulating angiogenesis and neurogenesis. ${ }^{8,9}$ Conversely, in vitro models have provided significant insight into the potential detrimental effects of miRNAs on signalling contributing to the development of ischaemic injury, such as miR-155 which has been shown to regulate reactive oxygen species production, nitric oxide generation and apoptosis in the endothelial cells. ${ }^{10}$ The silencing of miR-155 demonstrates neuroprotective effects in the ischaemic environment by enhancing proliferation, migration, and tube formation in human brain endothelial cells. In the setting of HS, miRNA overexpression has been shown to improve the brain blood barrier integrity, suppress inflammation and inhibit neuronal apoptosis, while other dysregulated miRNAs have the potential to alleviate neurological deficits via suppression of neuroinflammation and reduction of cell death. ${ }^{11-13}$
miRNAs represent a novel class of biomolecules with significant attractive properties centred around their ability to target multiple effectors within a signalling network; this is of particular importance given the heterogeneous nature of stroke pathophysiology. Circulating miRNAs are easily accessible and detectable in biofluids, and the identification of unique differential miRNA profiles in blood and urine places miRNAs in a unique position as diagnostic biomarkers for disease progression and measurement of therapeutic response. ${ }^{14-16}$ While much effort has been directed towards the identification of protein and metabolic biomarkers, as a result of the low sensitivity of proteins, there is currently no rapid diagnostic assay which would allow better management of acute stroke. The rapid induction of an ischaemic miRNA profile can be detected before the induction of protein markers, and their non-invasive, easy detection by quantitative polymerase chain reaction (qPCR)-based platforms provides rapid and cost-efficient profiling, capable of detecting even low-abundance miRNAs. Another advantage lies in their potential to act as a 'multifunctional' assay for diagnosis, informing treatment decisions and evaluating treatment response in the acute phase in contrast to protein biomarkers, where many protein markers would be required, with current analytical methods proving both expensive and time-consuming. While there remains a need for the identification of sensitive blood biomarkers that correlate with the development, diagnosis and prognosis of acute stroke, research centred around miRNAs as biomarkers in stroke is still in its early stages and the findings of such studies generally lack validity. The remarkable stability of miRNAs in biofluids, combined with their ease of access places circulating miRNAs in a promising position as minimally invasive clinical biomarkers in the diagnosis and prognosis of stroke. We here present a comprehensive review of the current clinical status and utility of circulating miRNAs in whole blood, plasma and serum for their ability to diagnose and assist in the prognosis of patients suffering stroke in the acute phase to better inform patient management and outcomes.

\section{Methods}

\section{Search strategy}

We carried out a systematic review in line with the Preferred Reporting Items for Systematic Reviews and Meta-Analyses (PRISMA) reporting guidelines. ${ }^{17}$ The protocol was not registered. In order to maximise our search, we combined Medical Subjective Heading (MeSH) terms with keyword terms to formulate the search strings. We conducted a comprehensive search of the databases Medline PubMed, Scopus, Cinahl, Cochrane, Web of Science, and Embase. Our MeSH and keyword 
search terms were combined as follows: (miRNA OR miRNAs OR microRNA OR microRNAs OR circulating miRNA OR circulating microRNAs OR miR) AND (Diagnostic OR diagnosis OR prognosis $O R$ prognostic $O R$ prediction) AND (Stroke OR acute stroke $O R$ ischaemic stroke $O R$ ischemic stroke $O R$ ischaemia $O R$ ischemia $O R$ haemorrhagic stroke $O R$ hemorrhagic stroke OR cerebrovascular accident OR cerebral infarction OR brain venous infarction). Our search strategy was defined in consultation with a senior medical librarian. All searches were restricted to the English language and no time limitation was applied.

\section{Inclusion and exclusion criteria}

We applied the following inclusion and exclusion criteria to our search. Inclusion criteria: (1) case-control studies; (2) image-based diagnosis of acute ischaemic and HS; (3) initial blood samples taken within the first 24 hours from onset of symptoms. Exclusion criteria: (1) animal model studies; (2) non-acute stroke studies; (3) article not available in English; (4) study does not present original data (letters; replies; commentaries; opinion articles); (5) non-blood biomarkers (e.g., cerebrospinal fluid). For papers which were not accessible online, authors were contacted and asked to provide a copy of their study. Papers were excluded from the review where studies were not accessible.

\section{Study selection and data extraction}

Following removal of duplicates, paper titles and abstracts were screened to identify relevant papers. Two authors independently applied the inclusion and exclusion criteria to all retrieved citations with arbitration by a third reviewer in the event of lack of consensus. Complete full-text analysis was then carried out on articles that were included following title and abstract screening for final inclusion. Reference lists of identified records were manually checked to identify additional relevant studies. For each study, two reviewers independently extracted and compared data on patient cohorts, sample type and time point from onset, miRNA selection, normalisation and quantification strategies, and 95\% confidence interval, area under the curve, sensitivity, and specificity of dysregulated miRNAs.

\section{Included studies quality assessment}

The quality of the included studies and risk of bias was assessed by the Newcastle-0ttawa Scale. ${ }^{18}$ Studies were scored according to quality of study group selection, comparability, and outcome. A final quality score was calculated for each study ranging from 0 to 9 . Studies were classified as high (7 to 9), medium (4 to 6), or low (0 to 3) quality.

\section{Bioinformatic miRNA gene target prediction and analysis}

Three miRNA gene target prediction tools (TargetScan v7.2, miRDB [accessed 17th July 2020], and miRWalk v3.0) were used to predict gene targets for miR-16-5p, miR-126-5p, and miR$335-5 p$, as miRNAs identified as having highest sensitivity and specificity with diagnostic and prognostic potential. Gene targets predicted by at least two of these tools were used for the gene ontology (GO) analyses. All GO enrichment analyses were carried out using EnrichR (version: January 23rd, 2019). An adjusted $P$-value cut-off of below 0.05 was used to filter results. The code used in the miRNA gene target prediction analyses and $\mathrm{GO}$ can be found at: https://github.com/GiantSpaceRobot/ miRNA-gene-target-prediction.

\section{Results}

\section{Included studies}

The initial comprehensive search of all the sources yielded 873 papers eligible for screening. After removal of 202 duplicates, 671 unique articles were assessed for inclusion by title and abstract screening, and a further 551 articles were excluded. Complete full-text analysis was carried out on the remaining 120

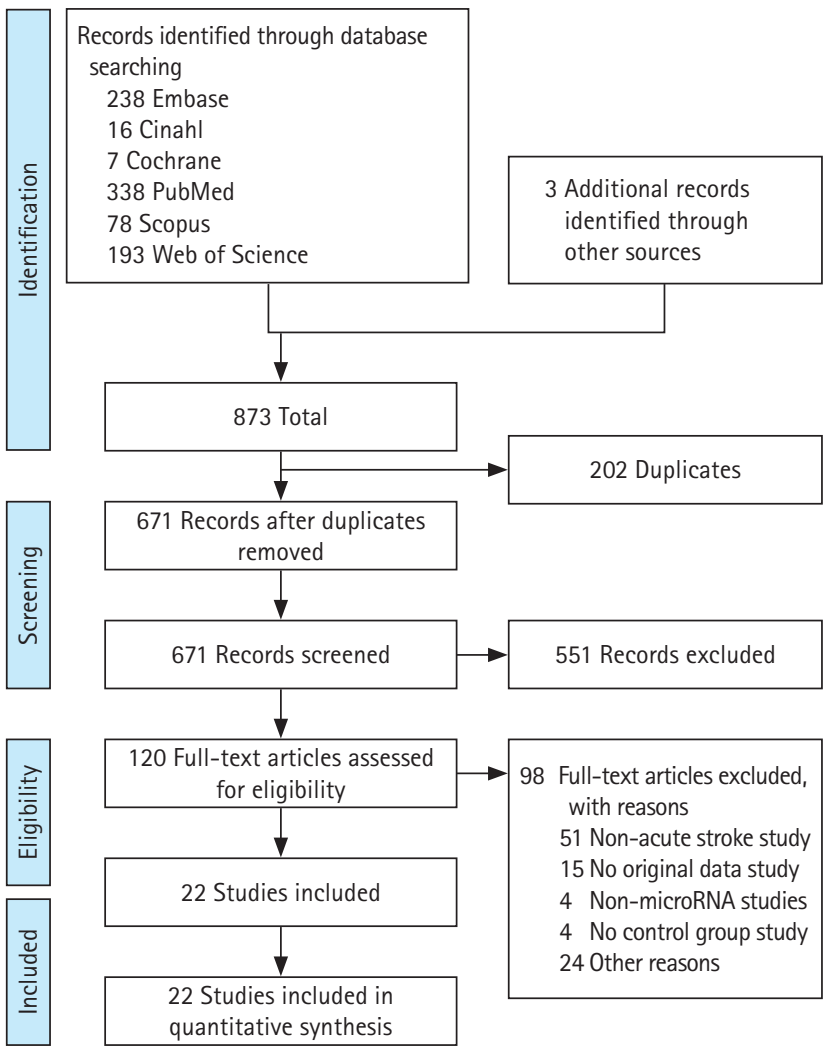

Figure 1. Flow chart detailing study screening and selection process. 
articles and finally 22 articles were included (Figure 1). Out of 22 case-controlled studies assessing acute stroke, a total of 22 IS and two HS cohorts were assessed, with a total of 2,470 AIS, $69 \mathrm{HS}$, and 1,651 healthy control (HC) subjects. One study also included transient ischaemic attack (TIA) patients, one recruited non-stroke patients with metabolic complications and one stratified AIS by treatment with recombinant tissue plasminogen activator. ${ }^{19-21}$ Five studies recruited less than 50 patients with the remainder recruiting 50 or more. ${ }^{22-26}$ Seven studies included only patients with a clinical diagnosis of first IS presentation, excluding recurrent IS patients (Table 1). ${ }^{22-24,27-30}$ Almost all studies excluded patients with a recent history of active infection and active cancer or treatment. Only eight studies classified AIS patients by Trial of Org 10172 in Acute Stroke Treatment (TOAST) criteria which subtypes stroke aetiology into cardioembolism, large artery atherosclerosis (LAA), small vessel disease, and undetermined cause. ${ }^{31}$ Included studies did not provide any information about the effect of age and sex on miRNA expression after stroke.

\section{miRNA profiling}

Eleven studies assessed miRNAs from plasma; ${ }^{19,21-23,25,27,30,32-35}$ seven studies from serum, ${ }^{, 22,26,36-40}$ and four assessed whole blood miRNAs. ${ }^{20,24,28,41}$ Six studies assessed plasma-extracted total RNA from blood collected using ethylenediaminetetraacetic acid (EDTA). Six studies utilized miRNA microarray to profile miRNA expression, while only one study employed small RNA sequencing techniques. The remaining studies selected their miRNAs based on evidence from previous work using in vivo or in vitro models or published in the existent literature at the time of the study. A mix of endogenous and synthetic controls were employed for normalisation. Eleven studies used endogenous U6 small nuclear RNA (snRNA), with a further three using RNU44, U75 or a combination of miR-16 and/or miR-451 as endogenous controls. Eight studies used exogenous cel-miR-39, celmiR-54, or UniSp2/UniSp4/UniSp5 mix (Exiqon) synthetic spikein controls. Twelve studies employed TaqMan miRNA Assay quantification, while the remaining studies employed SYBR Green-based methods for reverse transcription qPCR (RT-qPCR) analysis (Table 2).

\section{Diagnostic accuracy of identified miRNAs}

Diagnostic accuracy of specific miRNAs detected in the acute phase was assessed for sensitivity and specificity (Table 3). We identified miR-335 as demonstrating highest overall sensitivity in AIS (97.6\%), followed by miR-16 (94.7\%), while miR-16 also showed highest overall specificity (87\%) followed by miR-126 and let-7b (84\%) (Figure 2A). ${ }^{25,27,30,32}$ Upon assessment of stroke subtype, miR-16 was found to demonstrate the highest sensitivity (100\%) and specificity (91.3\%) in both large artery atherosclerosis stroke (LAAS) and total anterior circulation infarct (TACl) groups. ${ }^{27}$ miRNA-124-3p was reported as having a sensitivity of $68.4 \%$ and specificity of $71.2 \%$ in HS. $^{32}$

\section{Potential clinical utility of dysregulated miRNAs}

We define the clinical utility of the reported miRNAs based on their potential to diagnose and predict prognosis in acute stroke. The diagnostic potential was determined by the sensitivity and specificity of the reported miRNAs to correctly identify IS or HS stroke populations, while the prognostic potential was determined by the correlation of the reported miRNAs with clinical outcome such as the modified Rankin Scale (mRS) and National Institutes of Health Stroke Scale (NIHSS) (Table 3). We identified 33 dysregulated miRNAs demonstrating diagnostic potential in the acute phase of the disease (Figure 2B). We identified miR-16, miR-126, and miR-335 as having diagnostic and prognostic utility. miR-16 was reported to be upregulated by two studies, demonstrating both diagnostic and prognostic potential, with the ability to discriminate acute stroke subtype. ${ }^{25,32}$ Two studies, Leung et al. ${ }^{32}$ and Tian et al.. ${ }^{25}$ reported upregulation of miR-16 in AIS compared to HCs. Tian et al. ${ }^{25}$ reported that miR-16 was significantly upregulated in LAA and TACl subgroups of AIS. Leung et al. ${ }^{32}$ reported a correlation of miR-16 with mild stroke, defined as NIHSS $<5$ but there was no correlation with stroke volume measured radiologically. In comparison to Leung et al. ${ }_{1}^{32}$ Tian et al. ${ }^{25}$ reported that miR-16 expression was significantly higher in the poorer prognosis group (mRS 3-6) than in the good prognosis group (mRS 0-2). Downregulation of miR-126 was reported by two independent groups in three studies to have diagnostic, disease severity management, risk prediction, and monitoring potential. ${ }^{27,34,35} \mathrm{Jin}$ et al. ${ }^{35}$ reported a negative correlation between miR-126 expression and NIHSS score and pro-inflammatory cytokine elevation, placing it as biomarker of disease severity. miR-335 was reported by Zhao et al. ${ }^{30}$ as being downregulated and by using multiple logistic regression analysis it is suggestive that miR-335 can act as an independent risk factor for IS. Furthermore, miR-335 correlates negatively with NIHSS. Increased miR-130a was reported as a potential biomarker in early diagnosis of brain oedema and prognosis in $\mathrm{HS}$, correlating with perihematomal oedema severity, NIHSS, and mRS scores. ${ }^{36}$

\section{Bioinformatic miRNA gene target prediction and pathway enrichment}

Putative mRNA targets of miR-16-5p, miR-126-5p, and miR$335-5 p$, identified as having highest sensitivity and specificity 


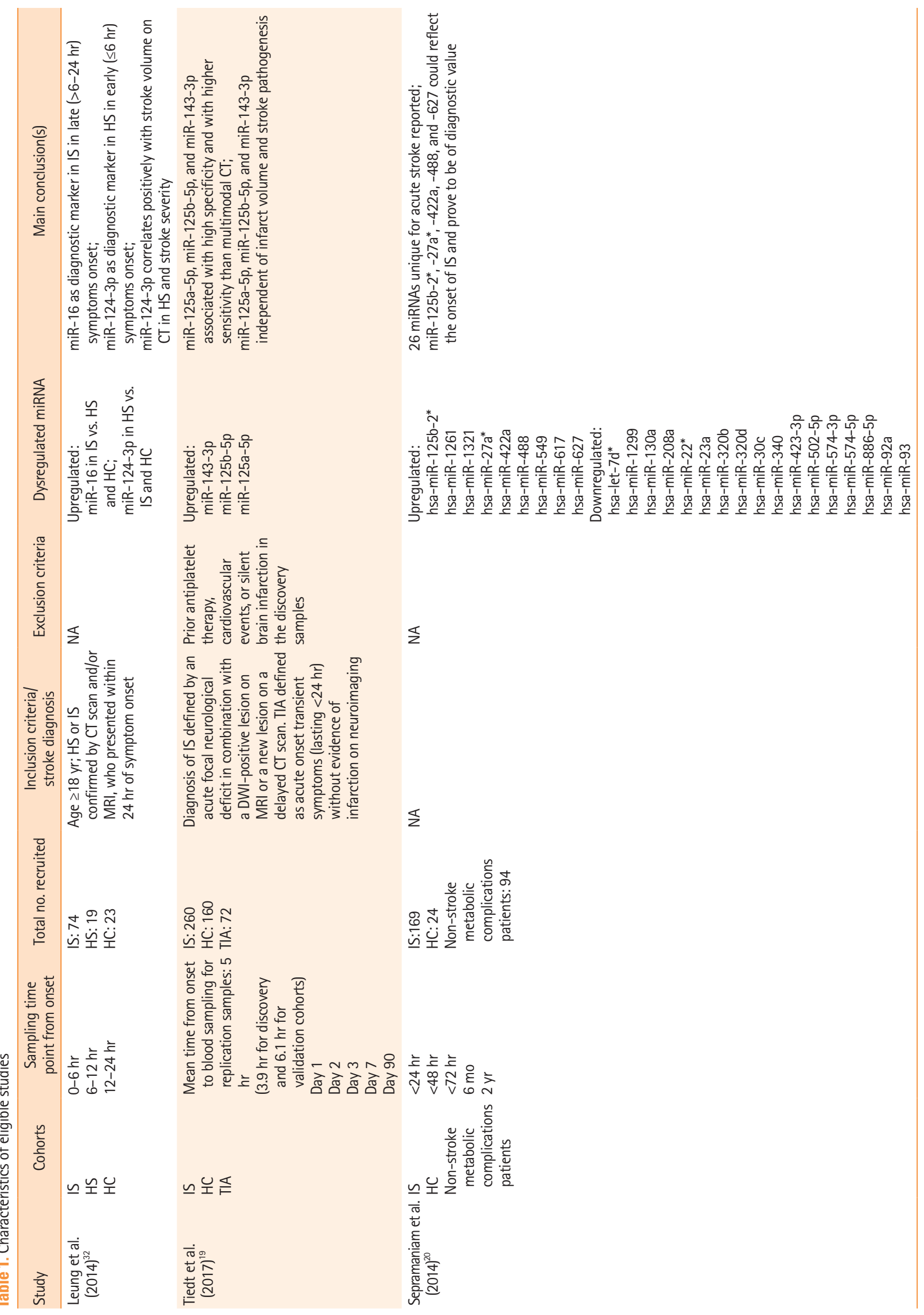




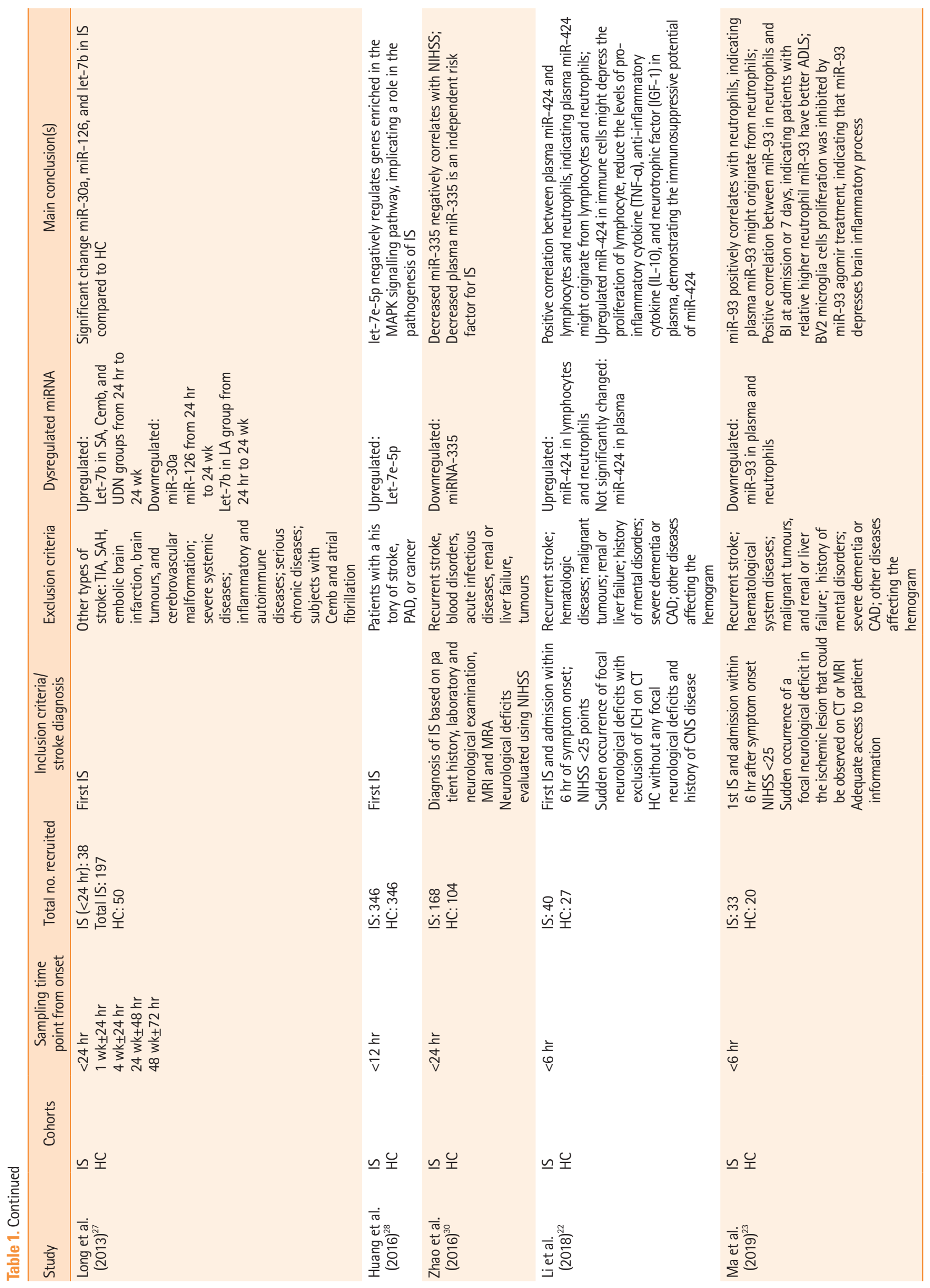




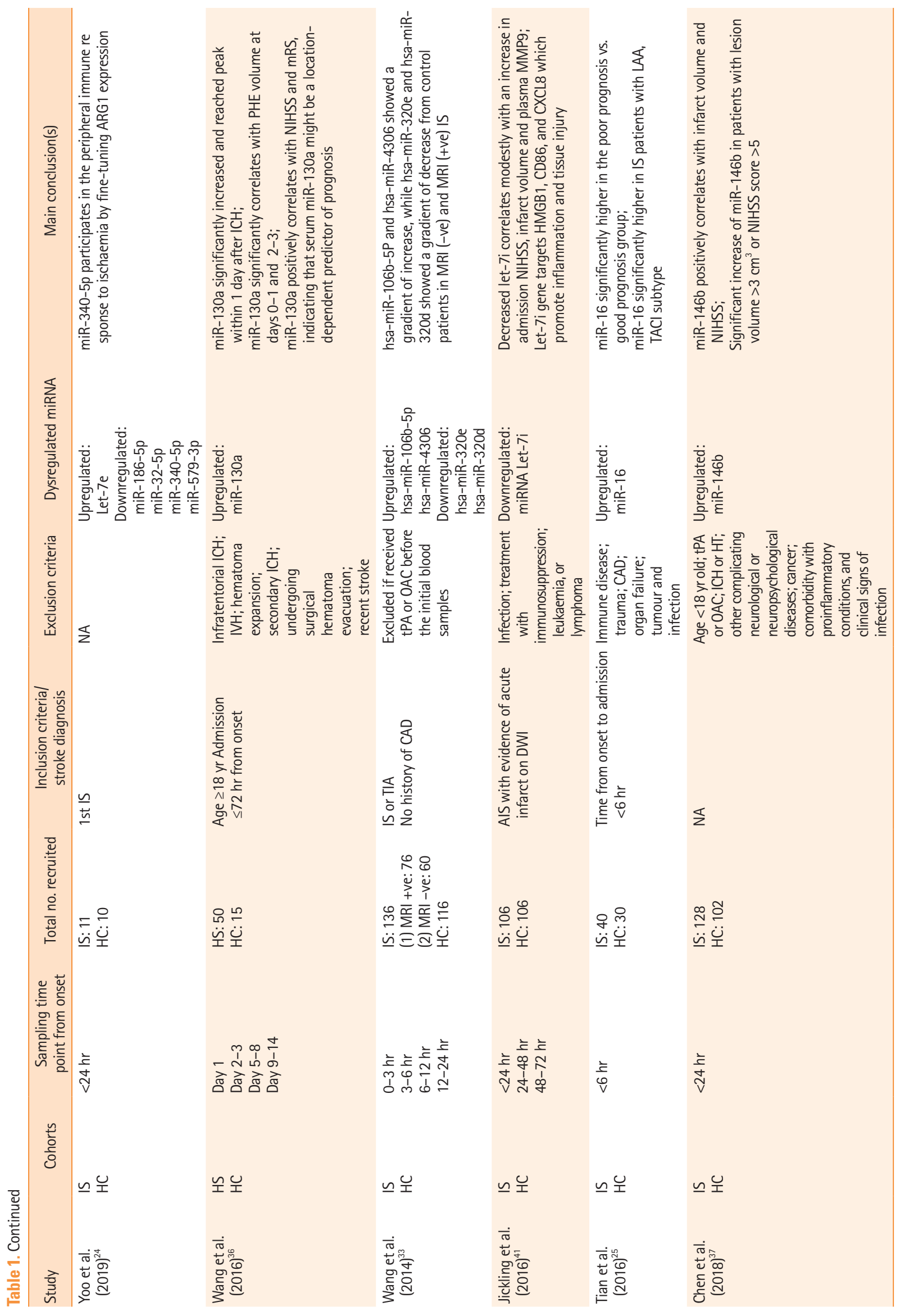




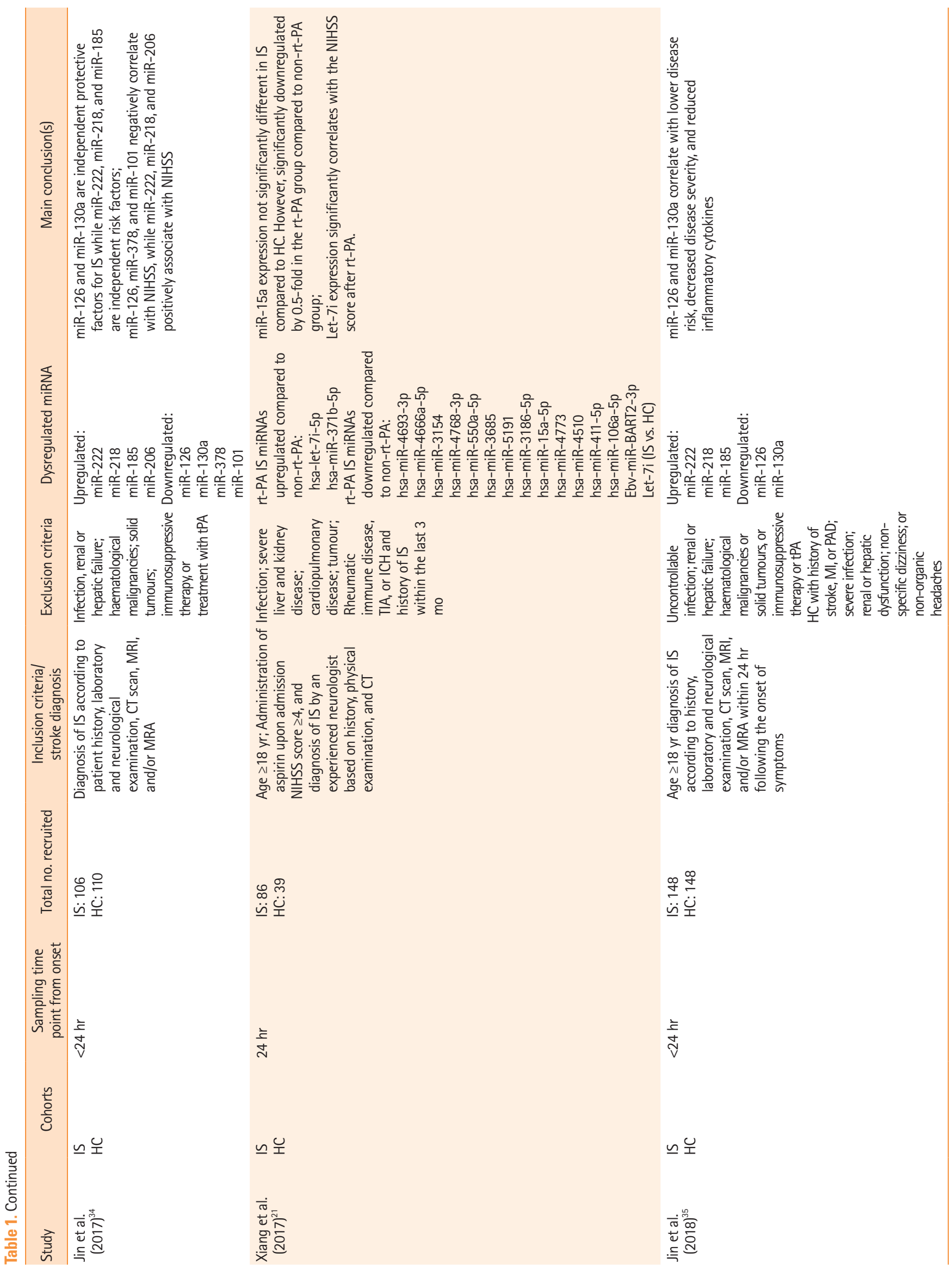




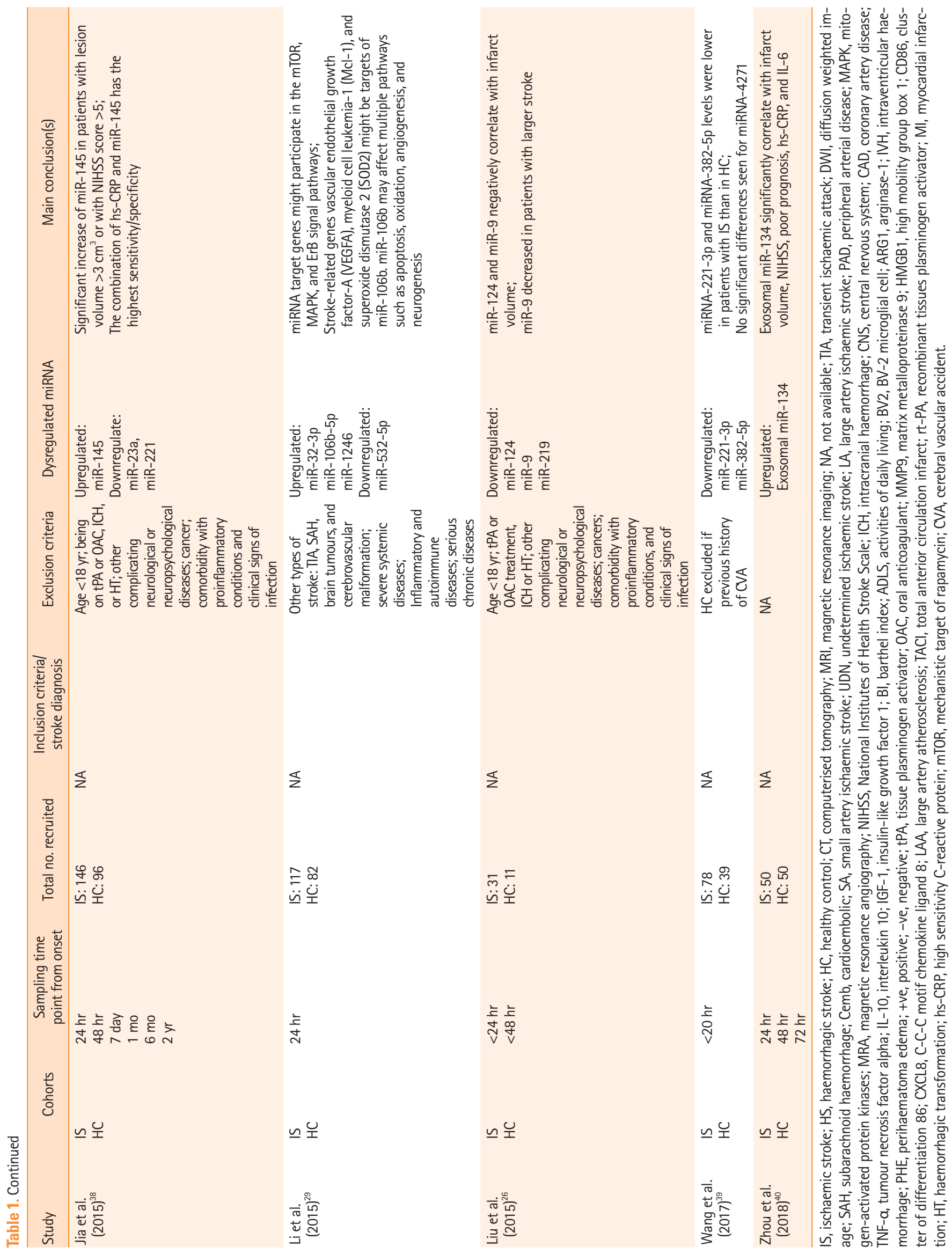



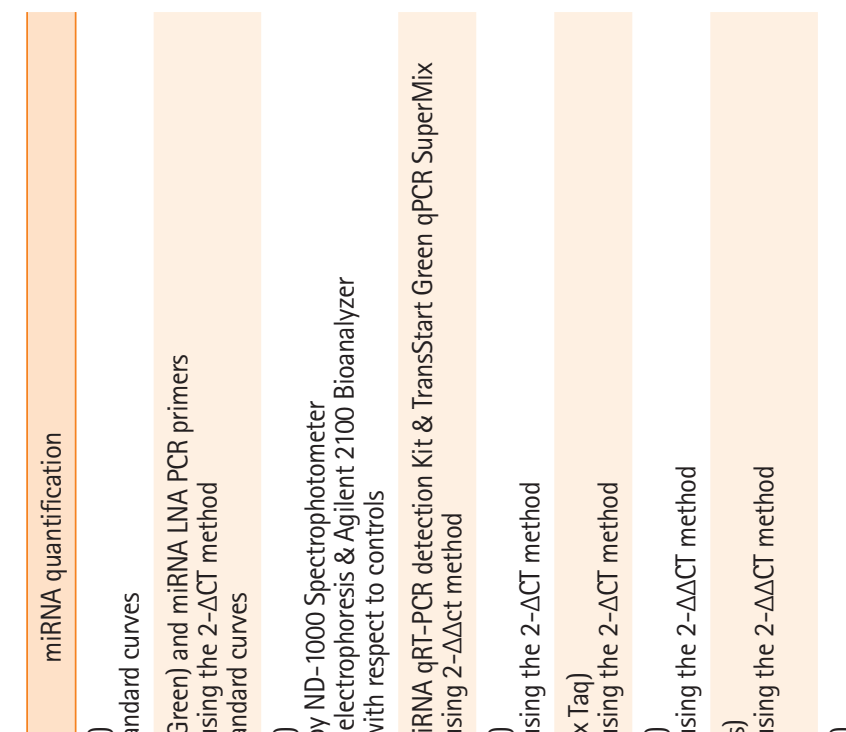


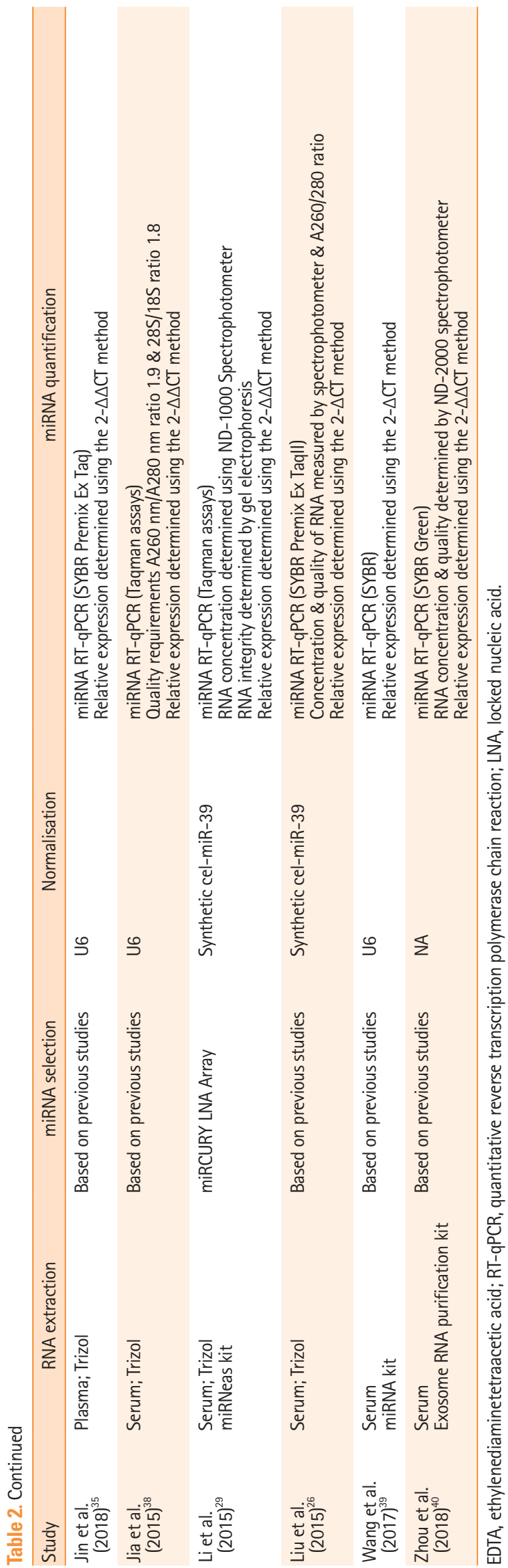

with diagnostic and prognostic potential, were identified using TargetScan, miRDB, and miRWalk. A total of 7,859 targets were predicted for miR-16-5p, 6,592 for miR-126-5p, and 1,171 for miR-335-5p. A total of 2,716 were predicted to be shared mRNA targets of both miR-16-5p and miR-126-5p, while 277 and 224 genes were shared targets of both miR-335-5p and miR-16-5p, and miR-335-5p and miR-126-5p respectively. A total of 376 genes were predicted to be targeted by all three miRNAs (Figure 3A).

We performed enrichment analysis of $\mathrm{GO}$ terms on predicted miRNA-target interactions (Figure $3 \mathrm{~B}$ and $\mathrm{C}$ ). The full results are available in Supplementary Table 2 (EnrichR_analysis Bejleri_2021). Our analysis of miR-16-5p targets revealed significant enrichment $(P$-adj $<0.05)$ of insulin signalling, Wnt signalling, transforming growth factor $\beta$ (TGF- $\beta$ ), mammalian target of rapamycin (mTOR), and mitogen-activated protein kinase (MAPK) signalling pathways. Signalling pathways such as these coordinate physiological and pathological processes in response to acute stroke, governing molecular activities at a cellular level. mRNA translation plays a pivotal role in the control of gene expression and regulation of homeostasis in response to disease, tightly controlled by signalling pathways in response to various stimuli, such as oxygen and nutrient deprivation and compromised energy status. Post-translational regulation, degradation or translation inhibition of mRNA highlights the important roles of miRNAs in response to disease pathophysiology. Our enrichment analysis indicates that increased miR-16 in response to ischaemia negatively regulates significantly high numbers of key proteins in the insulin and downstream mTOR and MAPK signalling pathways, impacting on the regulation of critical cellular responses including cellular metabolism, growth, proliferation, and homeostasis (Figure 4). Reported decreased expression of miR-126 following ischaemia carries significant implications for subsequent disinhibition of a significant number of predicted target genes associated with ErbB signalling, tumor necrosis factor (TNF) signalling, RAS signalling, and MAPK signalling pathways. Similarly, downregulation of miR-335 is associated with the positive regulation of predicted target Rho associated protein kinase 1 (ROCK1), involved in vascular inflammation, and NOTCH1 regulation of human endothelial cell calcification, linked to aortic valve calcification and stenosis, placing miR-126 and miR-335 as potential biomarkers of systemic inflammation. Pathways sharing common target genes predicted to be targeted by all three miRNAs are associated with MAPK signalling (13 common targets, $P<0.005)$, ErbB signalling ( 7 common targets, $P<0.005)$, insulin signalling ( 7 common targets, $P<0.05)$, and JAK-STAT signalling (8 common targets, $P<0.05$ ). 
Table 3. Diagnostic accuracy of reported miRNAs

\begin{tabular}{|c|c|c|c|c|c|c|c|}
\hline \multirow{3}{*}{$\begin{array}{l}\text { Study } \\
\text { Leung et al. } \\
(2014)^{32}\end{array}$} & \multicolumn{2}{|c|}{ miRNA } & \multirow{2}{*}{$\begin{array}{c}\text { Time point } \\
24 \mathrm{hr}\end{array}$} & \multirow{2}{*}{$\begin{array}{c}95 \% \mathrm{Cl} \\
0.59-0.79\end{array}$} & \multirow{2}{*}{$\begin{array}{l}\text { AUC } \\
0.70\end{array}$} & \multirow{2}{*}{$\begin{array}{c}\text { Sensitivity (\%) } \\
68.4\end{array}$} & \multirow{2}{*}{$\begin{array}{c}\text { Specificity }(\%) \\
71.2\end{array}$} \\
\hline & miR-124-3p & & & & & & \\
\hline & $\operatorname{miR}-16$ & & $24 \mathrm{hr}$ & $0.55-0.76$ & 0.66 & 94.7 & 35.1 \\
\hline Tiedt et al. $(2017)^{19}$ & $\begin{array}{r}\text { miR-125a-5p, } \\
\text { and miR-143 }\end{array}$ & $\begin{array}{l}n i R-125 b-5 p \\
3 p\end{array}$ & $<24 \mathrm{hr}$ & NA & 0.90 & 85.6 & 76.3 \\
\hline \multirow{6}{*}{$\begin{array}{l}\text { Sepramaniam et al. } \\
(2014)^{20}\end{array}$} & miR-125b-2 & & $24 \mathrm{hr}$ & $0.77-0.93$ & 0.85 & NA & NA \\
\hline & $\operatorname{miR}-27 a$ & & & $0.81-0.96$ & 0.86 & NA & NA \\
\hline & $\operatorname{miR}-422 a$ & & & $0.75-0.97$ & 0.86 & NA & NA \\
\hline & miR-488 & & & $0.72-0.92$ & 0.86 & NA & NA \\
\hline & miR-627 & & & $0.66-0.87$ & 0.76 & NA & NA \\
\hline & miR-920 & & & $1.00-1.00$ & 1.00 & NA & NA \\
\hline \multirow{3}{*}{$\begin{array}{l}\text { Long et al. } \\
(2013)^{27}\end{array}$} & miR-30a & & $24 \mathrm{hr}$ & $0.87-0.98$ & 0.91 & 94 & 80 \\
\hline & miR-126 & & $24 \mathrm{hr}$ & $0.87-0.98$ & 0.92 & 92 & 84 \\
\hline & Let-7b & & $24 \mathrm{hr}$ & $0.88-0.98$ & 0.93 & 92 & 84 \\
\hline $\begin{array}{l}\text { Huang et al. } \\
(2016)^{28}\end{array}$ & let-7e-5p & & $<12 \mathrm{hr}$ & $0.78-0.85$ & 0.82 & NA & NA \\
\hline $\begin{array}{l}\text { Zhao et al. } \\
(2016)^{30}\end{array}$ & miR-335 & & $<24 \mathrm{hr}$ & $0.86-0.93$ & 0.90 & 97.6 & 69.2 \\
\hline \multirow{8}{*}{$\begin{array}{l}\text { Wang et al. } \\
(2014)^{33}\end{array}$} & MRI-negative: & miR-106b-5p & $<24 \mathrm{hr}$ & $1.00-1.00$ & 1.00 & NA & NA \\
\hline & & miR-4306 & & $0.80-0.95$ & 0.88 & NA & NA \\
\hline & & miR-320e & & $0.91-0.99$ & 0.95 & NA & NA \\
\hline & & miR-320d & & $0.95-1.00$ & 0.98 & NA & NA \\
\hline & MRI-positive: & miR-106b-5p & $<24 \mathrm{hr}$ & $0.93-1.00$ & 0.96 & NA & NA \\
\hline & & miR-4306 & & $0.92-0.98$ & 0.95 & NA & NA \\
\hline & & miR-320e & & $0.96-1.00$ & 0.98 & NA & NA \\
\hline & & miR-320d & & $0.97-1.00$ & 0.99 & NA & NA \\
\hline \multirow{4}{*}{$\begin{array}{l}\text { Tian et al. } \\
(2016)^{25}\end{array}$} & miR-16 & & $<6 \mathrm{hr}$ & $0.65-0.90$ & 0.76 & 69.7 & 87 \\
\hline & miR-16 in LAA & & $<6 \mathrm{hr}$ & $0.88-1.02$ & 0.95 & 100 & 91.3 \\
\hline & miR-16 in TACl & & $<6 \mathrm{hr}$ & $0.93-1.03$ & 0.98 & 100 & 91.3 \\
\hline & miR-16 in PAC & & $<6 \mathrm{hr}$ & $0.64-0.95$ & 0.80 & 70.6 & 87 \\
\hline \multirow{4}{*}{$\begin{array}{l}\text { Chen et al. } \\
(2018)^{37}\end{array}$} & miR-146b & & $<24 \mathrm{hr}$ & $0.63-0.81$ & 0.78 & NA & NA \\
\hline & CRP \& miR-14 & & $<24 \mathrm{hr}$ & $0.80-0.94$ & 0.86 & NA & NA \\
\hline & IL-6 \& miR-14 & & $<24 \mathrm{hr}$ & $0.74-0.89$ & 0.82 & NA & NA \\
\hline & CRP \& IL-6 \& n & iR-146b & $<24 \mathrm{hr}$ & $0.80-0.93$ & 0.87 & NA & NA \\
\hline \multirow{6}{*}{$\begin{array}{l}\text { Jin et al. } \\
(2017)^{34}\end{array}$} & miR-126 & & $<24 \mathrm{hr}$ & $0.58-0.73$ & 0.65 & NA & NA \\
\hline & miR-130a & & $<24 \mathrm{hr}$ & $0.57-0.18$ & 0.64 & NA & NA \\
\hline & miR-222 & & $<24 \mathrm{hr}$ & $0.51-0.66$ & 0.58 & NA & NA \\
\hline & miR-218 & & $<24 \mathrm{hr}$ & $0.55-0.70$ & 0.62 & NA & NA \\
\hline & miR-185 & & $<24 \mathrm{hr}$ & $0.53-0.68$ & 0.60 & NA & NA \\
\hline & All 5 miRNAs c & mbined & $<24 \mathrm{hr}$ & $0.71-0.83$ & 0.77 & 87.7 & 54.5 \\
\hline \multirow{6}{*}{$\begin{array}{l}\text { Jin et al. } \\
(2018)^{35}\end{array}$} & $\mathrm{miR}-126$ & & $<24 \mathrm{hr}$ & $0.60-0.73$ & 0.67 & NA & NA \\
\hline & miR-130a & & $<24 \mathrm{hr}$ & $0.56-0.68$ & 0.62 & NA & NA \\
\hline & miR-222 & & $<24 \mathrm{hr}$ & $0.57-0.69$ & 0.63 & NA & NA \\
\hline & miR-218 & & $<24 \mathrm{hr}$ & $0.63-0.75$ & 0.69 & NA & NA \\
\hline & miR-185 & & $<24 \mathrm{hr}$ & $0.57-0.70$ & 0.63 & NA & NA \\
\hline & All 5 miRNAs c & mbined & $<24 \mathrm{hr}$ & $0.80-0.84$ & 0.84 & 83.8 & 69.6 \\
\hline
\end{tabular}


Table 3. Continued

\begin{tabular}{|c|c|c|c|c|c|c|}
\hline Study & miRNA & Time point & $95 \% \mathrm{Cl}$ & AUC & Sensitivity (\%) & Specificity (\%) \\
\hline \multirow{3}{*}{$\begin{array}{l}\text { Jia et al. } \\
(2015)^{38}\end{array}$} & CRP+miR-145 & $<24 \mathrm{hr}$ & $0.80-0.95$ & 0.90 & NA & NA \\
\hline & $C R P+m i R-23 a$ & $<24 \mathrm{hr}$ & $0.73-0.89$ & 0.82 & NA & NA \\
\hline & $C R P+m i R-221$ & $<24 \mathrm{hr}$ & $0.74-0.89$ & 0.82 & NA & NA \\
\hline $\begin{array}{l}\text { Liu et al. } \\
(2015)^{26}\end{array}$ & miR-124 & $<24 \mathrm{hr}$ & $0.61-0.92$ & 0.76 & NA & NA \\
\hline \multirow{3}{*}{$\begin{array}{l}\text { Wang et al. } \\
(2017)^{39}\end{array}$} & miR-221-3p & $<20 \mathrm{hr}$ & $0.73-0.90$ & 0.81 & NA & NA \\
\hline & miR-382-5p & $<20 \mathrm{hr}$ & $0.63-0.87$ & 0.75 & NA & NA \\
\hline & miR-4271 & $<20 \mathrm{hr}$ & $0.51-0.75$ & 0.63 & NA & NA \\
\hline
\end{tabular}

$\mathrm{Cl}$, confidence interval; $\mathrm{AUC}$, area under the curve; NA, not available; LAAS, large artery atherosclerosis stroke; TACl, total anterior circulation infarct; PACl, partial anterior circulation infarct; CRP, C-reactive protein.

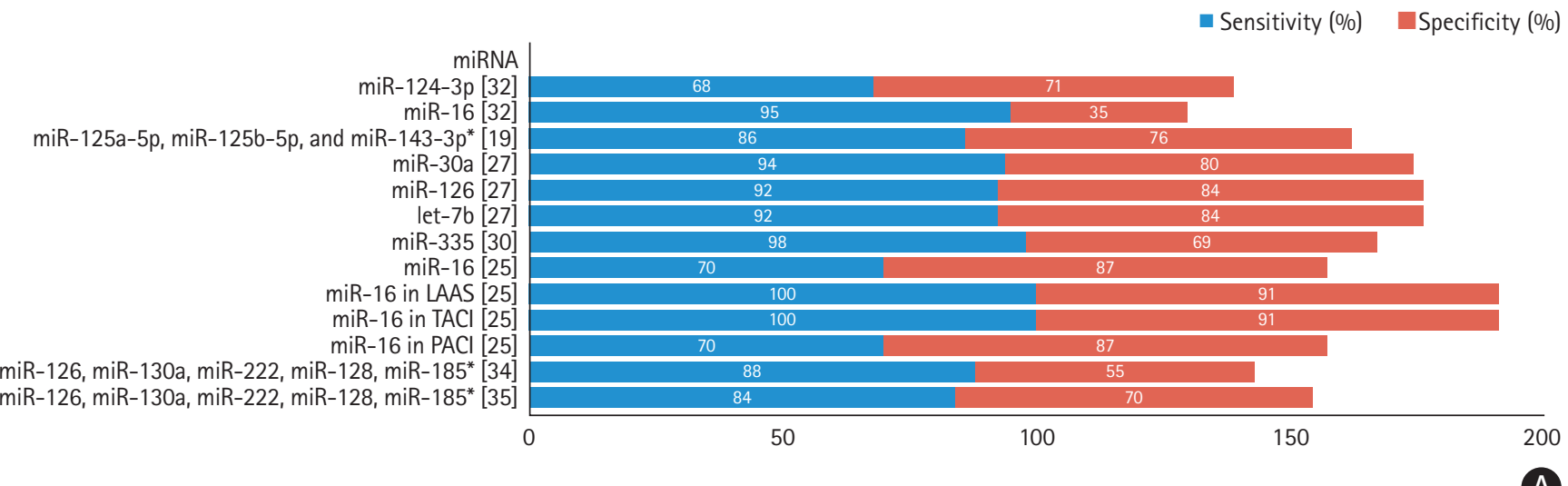

\begin{tabular}{|c|c|c|c|c|}
\hline \multicolumn{3}{|c|}{$\begin{array}{l}\text { Immunomodulators/ } \\
\text { neuroinflammation } \\
\downarrow \text { miR-124 } \downarrow \text { miR-9 } \\
\downarrow \text { miR-219 } \downarrow \text { miR-340-5p }\end{array}$} & & \multirow[t]{4}{*}{ Prognostic } \\
\hline & & & $\downarrow$ let-7i & \\
\hline \multicolumn{3}{|c|}{ Diagnostic } & \multirow{3}{*}{$\begin{array}{l}\uparrow \operatorname{miR}-16 \\
\uparrow \downarrow \text { miR-130a }\end{array}$} & \\
\hline $\begin{array}{l}\downarrow \text { miR-30a } \\
\downarrow \text { miR-320e } \\
\downarrow \text { miR-320d } \\
\downarrow \text { miR-23a } \\
\downarrow \text { miR-221 } \\
\downarrow \text { miR-532-5p }\end{array}$ & $\begin{array}{l}\uparrow \mathrm{miR}-124-3 p \\
\uparrow \mathrm{miR}-125 b-2 \\
\uparrow \mathrm{miR}-27 \mathrm{a} \\
\uparrow \mathrm{miR}-422 \mathrm{a} \\
\uparrow \mathrm{miR}-488 \\
\uparrow \mathrm{miR}-627\end{array}$ & $\begin{array}{l}\uparrow \text { miR-32-3p } \\
\uparrow \text { miR-1246 } \\
\uparrow \text { let-7e-5p } \\
\uparrow \text { miR-185 } \\
\uparrow \text { miR-125a-5p } \\
\uparrow \text { miR-125b-5p }\end{array}$ & & \\
\hline $\begin{array}{l}\downarrow \text { miR-335 } \\
\downarrow \text { miR-221-3p } \\
\downarrow \text { miR-382-5p }\end{array}$ & $\begin{array}{l}\uparrow \text { miR-106b-5p } \\
\uparrow \text { miR-4306 } \\
\uparrow \text { miR-145 }\end{array}$ & $\begin{array}{l}\uparrow \text { miR-143-3p } \\
\uparrow \text { miR-146b } \\
\uparrow \downarrow \text { let-7b }\end{array}$ & & \multirow[b]{3}{*}{$\begin{array}{l}\text { Disease risk prediction and } \\
\text { disease monitoring }\end{array}$} \\
\hline \multicolumn{3}{|c|}{$\uparrow \mathrm{miR}-222 \uparrow \mathrm{miR}-218$} & $\downarrow$ miR-126 & \\
\hline \multicolumn{3}{|c|}{$\begin{aligned} \text { Disease Severity Management } & \\
& \downarrow \text { miR-378 } \\
& \downarrow \text { miR-101 } \\
& \uparrow \text { miR-206 }\end{aligned}$} & & \\
\hline
\end{tabular}

B

Figure 2. Clinical utility of identified miRNAs in acute stroke. (A) Diagnostic accuracy of acutely expressed miRNAs within or at 24 hours of stroke symptoms onset. Study reference indicated in brackets. (B) Clinical utility of dysregulated miRNAs. LAAS, large artery atherosclerosis stroke; TACI, total anterior circulation infarct; PACl, partial anterior circulation infarct. *Denotes combination of miRNAs. 


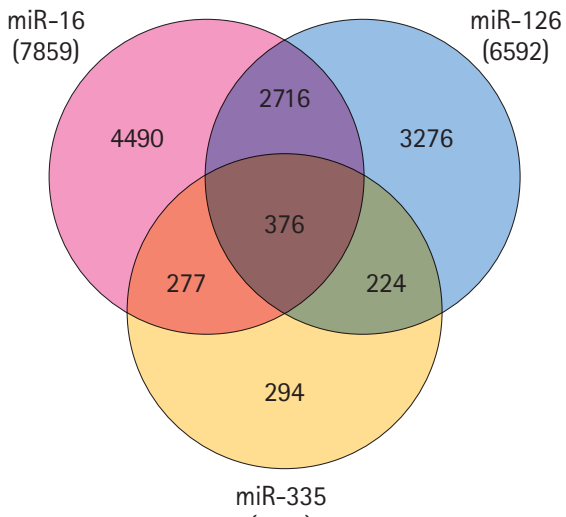

(1171)

A

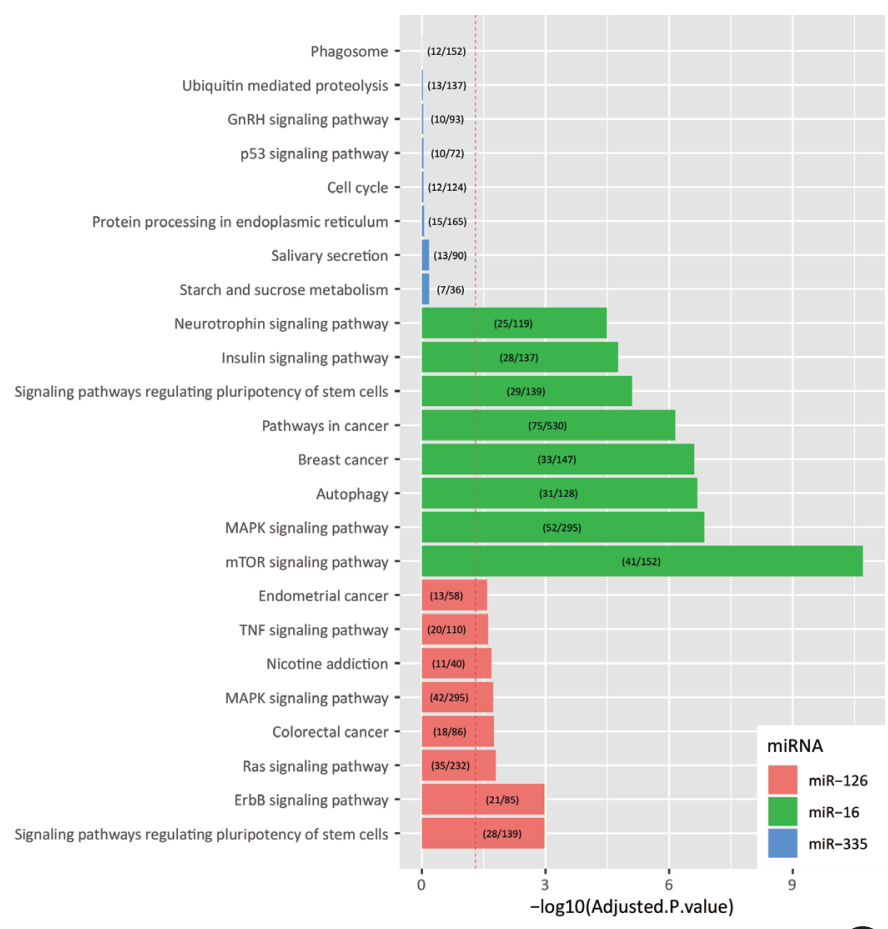

B

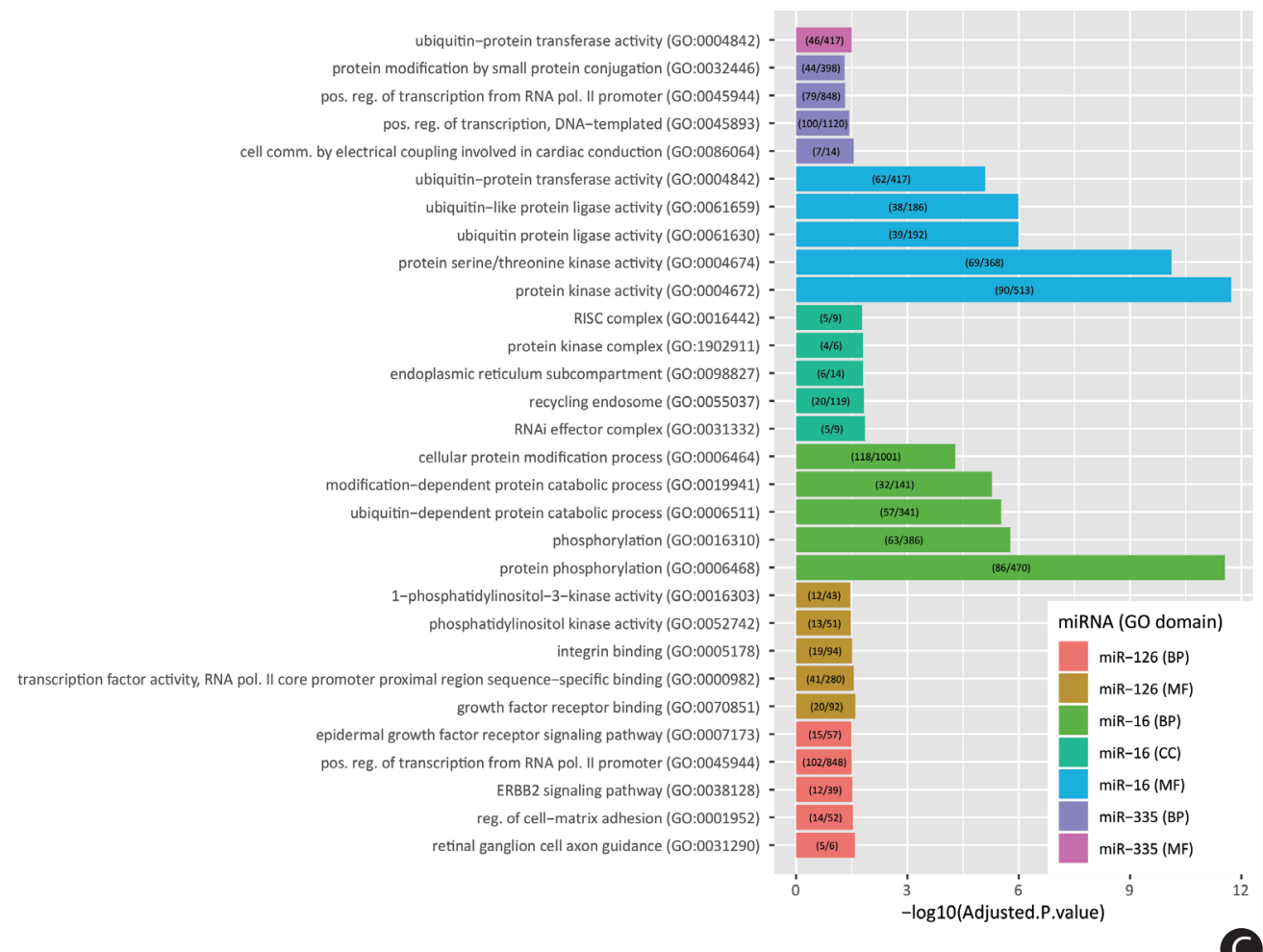

Figure 3. Bioinformatic miRNA gene target prediction and pathway enrichment. (A) Total and shared predicted mRNA targets of miR-16-5p, miR-126-5p, and miR-335-5p, identified as having highest sensitivity and specificity with diagnostic and prognostic potential. Barplots showing the top (B) Kyoto Encyclopedia of Genes and Genomes (KEGG) terms and (C), enriched gene ontology (GO) terms per group. The fraction in each bar represents the number of predicted gene targets/total number of genes associated with a given pathway or G0. Adjusted $P$ values (Benjamini-Hochberg corrected) $<0.05$ were considered significant. $\mathrm{GnRH}$, gonadotropin-releasing hormone; MAPK, mitogen-activated protein kinase; mTOR, mammalian target of rapamycin; TNF, tumor necrosis factor; ErbB, epidermal growth factor; RISC, RNA-induced silencing complex; MF, molecular function; BP, biological process; CC, cellular component. 


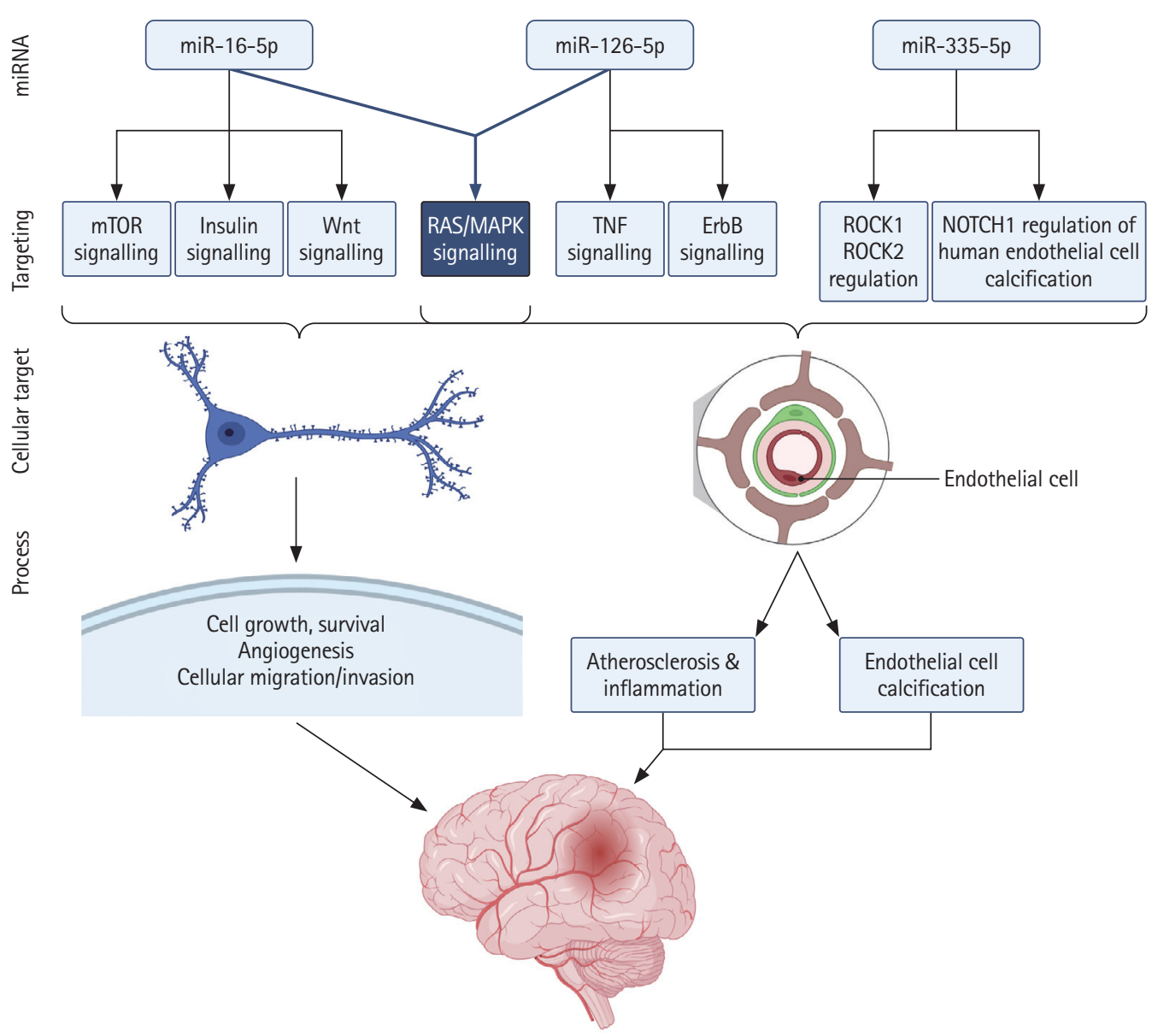

Figure 4. Potential roles for miR-16-5p, miR-126-5p, and miR-335-5p in the pathogenesis of ischaemic stroke. Putative targets for miR-16-5p were significantly enriched in mammalian target of rapamycin (mTOR), insulin, Wnt, and Ras/mitogen-activated protein kinases (MAPK) signalling pathways involved in regulation of roles central to neuronal survival and death and infarct development following stroke. Ras/MAPK is a common signalling pathway significantly enriched for the targets of both miR-16-5p and miR-126-5p. Also enriched in targets of miR-126-5p were tumor necrosis factor (TNF) and epidermal growth factor (ErbB) signalling pathways related to immune and inflammatory responses and endothelial cell growth, survival and proliferation. miR-335-5p regulation of Rho associated protein kinase 1 (ROCK1), involved in vascular inflammation, and NOTCH1 regulation of human endothelial cell calcification, is linked to aortic valve calcification and stenosis, common causes of cardiovascular morbidity and mortality. Figure produced in Biorender.

\section{Quality scores}

Quality scores for the included studies ranged from 2 to 9 (Supplementary Table 1). Three studies were classified as having low quality as they had a score $\leq 3{ }^{26,30,36}$ Eleven studies were classified as medium quality and eight studies as having high quality.

\section{Discussion}

We identified miR-16 and miR-126, reported by more than one study, and miR-335 as having the highest sensitivity as potential biomarkers with diagnostic and prognostic utility in AIS. miR-16 was reported to be upregulated in IS when compared to HS patients and HCs, with particularly high diagnostic capability in LAAS and TACl subgroups. The potential mechanisms of miR-16 in ischaemic reperfusion injury have been extensively studied in middle cerebral artery occlusion (MCAO) mice models and it has been shown that miR-16 promotes ischaemic brain injury by negatively regulating anti-apoptotic $\mathrm{BCl}-2$ and $\mathrm{Bcl}-\mathrm{w}^{42}$ Tian et al. ${ }^{25}$ demonstrated that miR-16 expression was markedly higher in stroke groups with poorer clinical outcomes (mRS 3-6) compared to those with a more favourable clinical outcome (mRS 0-2).

We report significant overrepresentation of several signal transduction networks by predicted targets of miR-16, notably insulin signalling and associated downstream mTOR and MAPK signalling pathways. Insulin-mediated signalling plays critical roles in the integration of various signals in response to environmental stress and nutrient availability and coordination of cellular response, and downstream activation of mTOR and RAS/MAPK signalling modulates cellular metabolism, growth, proliferation, and homeostasis. Of particular note in the context of our study, mTOR signalling is known to be dysregulated 
following ischaemia and oxygen-glucose deprivation (OGD), playing roles central to neuronal survival and death, and infarct development. Similarly, activation of MAPK signalling following IS is known to play roles in the regulation of neuronal survival, inflammation, and apoptosis. As a result, these signalling pathways have been the focus of a number of studies attempting to achieve neuroprotection in the treatment of IS, with current evidence indicating that the potential neuroprotective effects of key targets in these pathways do not function in isolation but as part of an integrated signalling response in relation to cellular survival, highlighting the potential importance of the multitargeting regulation of these pathways by miR-16 in the development of cerebral infarction. Interestingly, our analyses also revealed a relationship between miR-16 and Wnt signalling and significant enrichment of miR-16 targets in the TGF- $\beta$ signalling pathway. Wnt has been shown to be critically involved in neurogenesis and functional recovery following ischaemia while TGF- $\beta$ plays roles in mediating the angiogenic and immune response in ischaemic brain tissue. ${ }^{43}$ miR-16 is highly conserved and ubiquitously expressed across tissues and cell types, with well-characterised roles in the negative regulation of cell proliferation \& angiogenesis. ${ }^{44,45}$ Increased miR-16 following AIS, negatively regulating complex signal transduction networks identified here, carries significant implications for the regulation of fundamental biological processes critical for coordination of cellular response to ischaemia and associated excitotoxicity. Notably, the critical roles of insulin, mTOR, MAPK, and Wnt signalling have been a focus of significant interest as potential mechanisms affecting cellular survival and injury in the treatment of stroke. ${ }^{46,47}$

Three separate studies from two independent groups reported miR-126 as having diagnostic, disease risk prediction, monitoring, and management potential in AIS, demonstrating high sensitivity and specificity alone and in combination. ${ }^{27,34,35}$ miR-126 has been shown to be highly abundant and an endothelial-specific miRNA in capillaries and larger vessels, with key roles in vascular integrity and ischaemia-induced angiogenesis. ${ }^{48}$ Lower levels of miR-126 have been associated with increased plasma levels of the pro-angiogenic and pro-inflammatory mediator, placenta growth factor, a member of the vascular endothelial growth factor family and primary inflammatory instigator of atherosclerosis development and atherosclerotic plaque instability in coronary artery disease (CAD) and associated disease severity. ${ }^{49}$ Decreased endothelial progenitor cell expression of miR-126 has been reported in CAD, heart failure, and diabetic patients..$^{50}$ miR-126 has been reported as a potential biomarker of stroke severity as it negatively correlates with the NIHSS, a measure of stroke severity, and its association with cardiovascu- lar disease, immune and inflammatory responses. Plasma miR126 has been shown to negatively correlate with inflammatory cytokine TNF- $\alpha$, interleukin $1 \beta$ (IL-1 $\beta)$, and IL-6 levels, while miR-126 over-expression in endothelial cells has been shown to decrease pro-inflammatory TNF- $a$ and high-mobility group box 1 (HMGB1), involved in the pathogenesis of IS and reperfusion injury. ${ }^{35,51}$ Our analysis also identified significant overrepresentation of miR-126-5p target genes associated with both TNF and RAS/MAPK signalling, and ErbB signalling, with significant roles, in microglia activation, cytokine production and inflammation, and endothelial cell proliferation and function. ${ }^{52}$ Decreased miR-126 and associated positive regulation of target genes in these pathways carries implications for dysregulation of acute inflammatory response and associated stroke pathology, further supporting a possible role for miR-126 as an endothelial cell-specific biomarker of systemic inflammatory status, damage, and severity. This is of particular interest given the strong association of stroke with hypertension and related cardiovascular diseases and associated poor prognosis and mortality.

miR-335 was found to have the highest overall sensitivity (98\%) amongst all miRNAs reported, and negative correlation with NIHSS, demonstrating promising diagnostic utility. miR335 has been reported to be decreased in rat MCAO models of AIS, and synthetic increased expression inhibits apoptotic signalling and reduces ischaemic injury via downregulation of ROCK2. ${ }^{53}$ ROCK1 and ROCK2 are both reported targets of miR-335, involved in fundamental cellular functions including vascular inflammation, cell adhesion, migration, cytoskeleton organization, and stress fibre formation. Our analysis identified ROCK1 as a predicted target of miR-335-5p, and enrichment of miR-335-5p target genes in apoptosis modulation and signalling. We also identified a number of predicted miR-335-5p target genes in NOTCH1 regulation of human endothelial cell calcification. Dysregulation of NOTCH signalling is increasingly linked to aortic valve calcification, a leading cause of heart disease, and dysregulation of miR-335 has also been reported in a mouse model of aortic valve stenosis, supporting a role for miR335 specificity in patients suffering AIS. ${ }^{54}$

Our analysis highlights significant roles for miR-16, miR-126, and miR-335 in the modulation of pathways involved in stroke pathophysiology, immunomodulation and inflammation regulation. It should be emphasized that the functional consequences of miRNA modulation on the identified pathways are dynamic, and both the consequences of the activated signalling pathways and of miRNA regulation of targets are cell- and disease-context dependent. The multi-targeting abilities of miRNAs exert an effect at a systems level in response to environmental and intra-cellular cues, and the impact of target pathway deregula- 
tion is dependent on the duration as well as the severity of the insult, such as OGD. Of particular note, we have here identified a significantly high number of gene targets of these ischaemia-associated miRNAs involved in insulin, MAPK, mTOR, and Wnt signalling pathways, carrying significant implications for infarct development and expansion in IS (Figure 4). Elucidation of the mechanisms underlying the pathophysiology of IS represents a promising approach to the development of therapeutic strategies which can halt the progression and expansion of cell death, and promote neuroprotection in acute ischaemia. We identified a small number of common targets of all three miRNAs, involved in the co-ordination of the cellular stress response to ischaemic environment, with impacts on salvageable tissue. Increased sensitivity (100\%) and specificity (91.3\%) of miR-16 in both LAAS and TACl groups, compared to partial anterior circulation infarct (70.6\% and 87\% respectively), supports a diagnostic and prognostic role in the severity of compromised tissue. Similarly, decreased miR-126 and miR-335 expression and their association with cardiovascular disorders and regulation of immune and inflammatory responses supports their significant sensitivity and specificity as biomarkers of systemic inflammatory status, damage and severity in IS. In addition to their diagnostic potential in AIS, dysregulation of these miRNAs presents promising utility providing information about underlying aetiology.

Of the identified studies, only one reported significant utility for miRNAs in HS. Wang et al. ${ }^{55}$ reported upregulation of miR130a in haemorrhagic patients and its potential use as a biomarker in the diagnosis of early oedema and prognosis in acute HS. Evidence in both in vitro and in vivo models has demonstrated that delivery of a miR-130a antagonist (antagomir) successfully attenuate brain oedema, reduce both blood-brain barrier permeability and infarct volume, and improve neurologic function. ${ }^{55}$

While the utility of circulating miRNAs as biomarkers has been recognised, several factors present problems in their successful translation and development in stroke. We identified wide variation in the number of patients and control groups recruited to each study. However, the diagnostic and prognostic utility of the miRNAs that we have reported were based on robust numbers of recruited patients with a mean cohort 10 number of 74 (range, 38 to 136$){ }^{25,27,32-34,36}$ Furthermore, 20 of the included studies were performed in Asia, one in Europe and one in USA, indicating that miRNA studies reported may not be representative of all cohorts and population differences in small non-coding RNA expression should be taken into consideration in translational study design. ${ }^{56}$ Varied inclusion and exclusion criteria in participant recruitment and sampling time of blood can contribute considerable variability given the rapid evolution of the miRNA profile and the heterogeneity of the developing ischaemic environment. Five studies included only first-time stroke patients or excluded recurrent stroke patients, while four studies excluded patients who had received thrombolytic therapy prior to the blood collection. There is now evidence that tissue plasminogen activator alters miRNA profile expression and subsequently the identification of diagnostic and prognostic biomarkers. ${ }^{21}$ All these confounding factors have the potential to impact detection of differential expression of miRNA profiles among the studies and warrant consideration in future study design.

Variability in the methodology used in miRNA studies from blood collection to miRNA profile discovery also warrants consideration. The most common sample type was plasma, followed by serum and a small number of studies employed whole blood. Recent evidence has shown a marked heterogeneity in miRNA expression from serum and plasma samples, impacting significantly on reporting of variances in miRNA profiling. ${ }^{57}$ The use of EDTA tubes from six studies adds more potential variability, as anti-coagulants such as heparin and EDTA are potent inhibitors of down-stream PCR reactions, impacting on the quality of down-stream analysis for biomarker identification. ${ }^{58}$ These differences in blood collection procedures can affect the coagulation cascade and, along with degree of haemolysis and platelet, red and white blood cell contamination, may influence the biochemical profiles.

Importantly, the most common control for miRNA normalization was endogenous U6, with other studies utilising endogenous miR-16, miR-451, RNU44, and U75. Just eight studies employed exogenous spike-in controls for normalisation. The use of snRNAs, such as U6, may not reflect the biochemical transcription, processing, and expression patterns of other RNA classes and as such may not be suitable for miRNA normalisation. Furthermore, serum levels of U6 have been reported to demonstrate a high variability in patients with critical illness, and to correlate with serum levels of established inflammatory biomarkers. ${ }^{59}$ Use of circulating miR-16 and miR-451 as a normalisation strategy, known to be significantly altered in plasma and serum in response to haemolysis, for blood samples from patients with acute intracerebral haemorrhage by one study in this review highlights the problematic area of lack of consensus on a normalisation strategy, particularly in the case of a haemolytic-susceptible circulating diagnostic or prognostic biomarker such as miR-16. However, due to the sensitivity and specificity of miRNA profiles in response to disease states, especially given the heterogeneous nature and aetiology of IS, the identification of a single reference with universal applicability will be difficult. To this end, the use of exogenous synthetic spiked-in miRNA during RNA isolation for normalisation represents a robust approach. 
The considerable variation in the pre-analytical and analytical approaches highlighted here represent a significant barrier in the identification of clinically useful miRNAs in translational stroke research, and are most likely responsible for the heterogeneous results yielded from our analysis and the number of studies that have reported the top three miRNAs which we have identified as having diagnostic and prognostic potential. The small number of studies which report these miRNAs raises concerns regarding the reproducibility of such studies. However, our bioinformatic analysis has demonstrated the pivotal role that these miRNAs play in stroke pathophysiology. The variability across profiling platforms, and in particular the lack of consensus on normalisation strategies confers a significant impact on the ability to detect significant biological differences across samples is such a heterogeneous disease setting, and therefore their utility in a clinical and research setting. Nonetheless, we present a comprehensive and encouraging review of current evidence on the current position of miRNAs as biomarkers of acute stroke and our analysis indicate the need for further research in order to confirm our findings and provide more evidence of the potential clinical utility of miRNAs in the field of translational stroke research.

\section{Conclusions}

We identified candidate miRNAs miR-16, miR-126, and miR335 as promising biomarkers for stroke with diagnostic and prognostic potential. Considerable variation in pre-analytical and analytical parameters which potentially contribute to the lack of overall consensus in miRNA expression profiles identified in this review highlights the need for internationally accepted and unified methodologies which would standardise the process of blood sampling, handling and preparation for miRNA screening, profiling and validation. It is important to acknowledge the potential contributions of underlying pathologies to miRNA dysregulation in the setting of AIS, as we have highlighted in our bioinformatic analyses. Inflammatory response has been well established as a biomarker of stroke, and miRNA changes may reflect the underlying aetiology and systemic inflammatory responses as factors related and consequential to AIS pathogenesis. While considerable advancements have been made in the development of miRNAs as therapeutic targets in nonstroke pathologies, the heterogeneity of stroke pathogenesis and associated biochemical cascades requires more robust investigation for the meaningful translation of miRNAs as therapeutic modulators in AIS. While the capacity of miRNAs to target multiple genes in multiple pathways places them in a unique position for development in AIS, the complexity of stroke pathophysiology and associated, equally complex, comorbidities requires greater consideration in the development of candidate miRNAs as biomarkers and potential therapeutic targets. Consideration for toxicity and off-target downstream effects resulting from the delivery of miRNA mimics and antagomirs highlights the need for robust pre-clinical development for the refinement and successful translation of miRNAs as multi-targeting biomarkers and promising therapeutic targets in AIS.

\section{Supplementary materials}

Supplementary materials related to this article can be found online at https://doi.org/10.5853/jos.2020.05085.

\section{Disclosure}

The authors have no financial conflicts of interest.

\section{Acknowledgments}

The authors acknowledge support by the RCSI StAR MD programme and Paul J. Murphy, Information Specialist, for his assistance with the systematic review.

\section{References}

1. GBD 2016 Stroke Collaborators. Global, regional, and national burden of stroke, 1990-2016: a systematic analysis for the Global Burden of Disease Study 2016. Lancet Neurol 2019;18:439-458.

2. Saenger $A K$, Christenson RH. Stroke biomarkers: progress and challenges for diagnosis, prognosis, differentiation, and treatment. Clin Chem 2010;56:21-33.

3. Dirnagl U, ladecola C, Moskowitz MA. Pathobiology of ischaemic stroke: an integrated view. Trends Neurosci 1999;22: 391-397.

4. Pfeiffer S, Anilkumar U, Chen G, Ramírez-Peinado S, Galindo-Moreno J, Muñoz-Pinedo $\mathrm{C}$, et al. Analysis of BH3-only proteins upregulated in response to oxygen/glucose deprivation in cortical neurons identifies Bmf but not Noxa as potential mediator of neuronal injury. Cell Death Dis 2014;5: e1456.

5. Whiteley W, Tseng MC, Sandercock P. Blood biomarkers in the diagnosis of ischemic stroke: a systematic review. Stroke 2008;39:2902-2909.

6. Hutchison ER, Okun E, Mattson MP. The therapeutic potential of microRNAs in nervous system damage, degeneration, and repair. Neuromolecular Med 2009;11:153-161. 
7. Zhang C. MicroRNomics: a newly emerging approach for disease biology. Physiol Genomics 2008;33:139-147.

8. Zhao H, Wang J, Gao L, Wang R, Liu X, Gao Z, et al. MiRNA-424 protects against permanent focal cerebral ischemia injury in mice involving suppressing microglia activation. Stroke 2013:44:1706-1713.

9. Zeng $L$, He $X$, Wang $Y$, Tang $Y$, Zheng $C$, Cai $H$, et al. MicroRNA-210 overexpression induces angiogenesis and neurogenesis in the normal adult mouse brain. Gene Ther 2014; 21:37-43.

10. Liu Y, Pan O, Zhao Y, He C, Bi K, Chen Y, et al. MicroRNA-155 regulates ROS production, NO generation, apoptosis and multiple functions of human brain microvessel endothelial cells under physiological and pathological conditions. J Cell Biochem 2015;116:2870-2881.

11. Zhang $Y$, Han B, He Y, Li D, Ma X, Liu Q, et al. MicroRNA-132 attenuates neurobehavioral and neuropathological changes associated with intracerebral hemorrhage in mice. Neurochem Int 2017;107:182-190.

12. Fu $X$, Niu T, Li X. MicroRNA-126-3p attenuates intracerebral hemorrhage-induced blood-brain barrier disruption by regulating VCAM-1 expression. Front Neurosci 2019;13:866.

13. Yuan B, Shen H, Lin L, Su T, Zhong L, Yang Z. MicroRNA367 negatively regulates the inflammatory response of microglia by targeting IRAK4 in intracerebral hemorrhage. J Neuroinflammation 2015;12:206

14. Pfeiffer $S$, Sánchez-Lechuga $B$, Donovan $P$, Halang L, Prehn JHM, Campos-Caro A, et al. Circulating miR-330-3p in late pregnancy is associated with pregnancy outcomes among lean women with GDM. Sci Rep 2020;10:908.

15. Raoof $R$, Jimenez-Mateos EM, Bauer S, Tackenberg B, Rosenow F, Lang J, et al. Cerebrospinal fluid microRNAs are potential biomarkers of temporal lobe epilepsy and status epilepticus. Sci Rep 2017;7:3328.

16. Bacon S, Engelbrecht B, Schmid J, Pfeiffer S, Gallagher R, McCarthy $A$, et al. MicroRNA-224 is readily detectable in urine of individuals with diabetes mellitus and is a potential indicator of beta-cell demise. Genes (Basel) 2015;6:399-416.

17. Moher D, Liberati A, Tetzlaff J, Altman DG; PRISMA Group. Preferred reporting items for systematic reviews and meta-analyses: the PRISMA statement. PLoS Med 2009;6: e1000097.

18. Lo CK, Mertz D, Loeb M. Newcastle-Ottawa Scale: comparing reviewers' to authors' assessments. BMC Med Res Methodol 2014;14:45.

19. Tiedt $S$, Prestel $M$, Malik $R$, Schieferdecker $N$, Duering $M$,

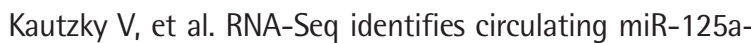
$5 p$, miR-125b-5p, and miR-143-3p as potential biomarkers for acute ischemic stroke. Circ Res 2017;121:970-980.

20. Sepramaniam S, Tan JR, Tan KS, DeSilva DA, Tavintharan S, Woon FP, et al. Circulating microRNAs as biomarkers of acute stroke. Int J Mol Sci 2014;15:1418-1432.

21. Xiang $W$, Tian $C$, Lin J, Wu X, Pang G, Zhou L, et al. Plasma let-7i and miR-15a expression are associated with the effect of recombinant tissue plasminogen activator treatment in acute ischemic stroke patients. Thromb Res 2017;158: 121-125.

22. Li G, Ma $\mathrm{Q}$, Wang R, Fan Z, Tao Z, Liu P, et al. Diagnostic and immunosuppressive potential of elevated Mir-424 levels in circulating immune cells of ischemic stroke patients. Aging Dis 2018;9:172-181.

23. Ma $\mathrm{Q}$, Li G, Tao Z, Wang J, Wang R, Liu P, et al. Blood microRNA-93 as an indicator for diagnosis and prediction of functional recovery of acute stroke patients. J Clin Neurosci 2019;62:121-127.

24. Yoo H, Kim J, Lee AR, Lee JM, Kim OJ, Kim JK, et al. Alteration of microRNA 340-5p and arginase-1 expression in peripheral blood cells during acute ischemic stroke. Mol Neurobiol 2019;56:3211-3221.

25. Tian C, Li Z, Yang Z, Huang $\mathrm{Q}$, Liu J, Hong B. Plasma microRNA-16 is a biomarker for diagnosis, stratification, and prognosis of hyperacute cerebral infarction. PLoS One 2016;11: e0166688.

26. Liu Y, Zhang J, Han R, Liu H, Sun D, Liu X. Downregulation of serum brain specific microRNA is associated with inflammation and infarct volume in acute ischemic stroke. J Clin Neurosci 2015;22:291-295.

27. Long G, Wang F, Li H, Yin Z, Sandip C, Lou Y, et al. Circulating miR-30a, miR-126 and let-7b as biomarker for ischemic stroke in humans. BMC Neurol 2013;13:178.

28. Huang S, Lv Z, Guo Y, Li L, Zhang Y, Zhou L, et al. Identification of blood let-7e-5p as a biomarker for ischemic stroke. PLoS One 2016;11:e0163951.

29. Li P, Teng F, Gao F, Zhang M, Wu J, Zhang C. Identification of circulating microRNAs as potential biomarkers for detecting acute ischemic stroke. Cell Mol Neurobiol 2015;35: 433-447.

30. Zhao B, Zhu Z, Hao J, Wan Z, Guo X. Decreased plasma miR335 expression in patients with acute ischemic stroke and its association with calmodulin expression. J Int Med Res 2016;44:1331-1338.

31. Adams HP Jr, Bendixen BH, Kappelle $\sqcup$, Biller J, Love BB, Gordon DL, et al. Classification of subtype of acute ischemic stroke. Definitions for use in a multicenter clinical trial. TOAST. Trial of Org 10172 in Acute Stroke Treatment. Stroke 1993;24:35-41. 
32. Leung $L Y$, Chan $C P$, Leung $Y K_{\text {, Jiang } H L}$, Abrigo JM, Wang de $F$, et al. Comparison of miR-124-3p and miR-16 for early diagnosis of hemorrhagic and ischemic stroke. Clin Chim Acta 2014;433:139-144.

33. Wang W, Sun G, Zhang L, Shi L, Zeng Y. Circulating microRNAs as novel potential biomarkers for early diagnosis of acute stroke in humans. J Stroke Cerebrovasc Dis 2014;23: 2607-2613.

34. Jin F, Xing J. Circulating pro-angiogenic and anti-angiogenic microRNA expressions in patients with acute ischemic stroke and their association with disease severity. Neurol Sci 2017;38:2015-2023.

35. Jin F, Xing J. Circulating miR-126 and miR-130a levels correlate with lower disease risk, disease severity, and reduced inflammatory cytokine levels in acute ischemic stroke patients. Neurol Sci 2018;39:1757-1765.

36. Wang MD, Wang Y, Xia YP, Dai JW, Gao L, Wang SQ, et al. High serum miR-130a levels are associated with severe perihematomal edema and predict adverse outcome in acute ICH. Mol Neurobiol 2016;53:1310-1321.

37. Chen Z, Wang K, Huang J, Zheng G, Lv Y, Luo N, et al. Upregulated serum miR-146b serves as a biomarker for acute ischemic stroke. Cell Physiol Biochem 2018;45:397-405.

38. Jia L, Hao F, Wang W, Qu Y. Circulating miR-145 is associated with plasma high-sensitivity C-reactive protein in acute ischemic stroke patients. Cell Biochem Funct 2015;33:314319.

39. Wang $Y, M a Z$, Kan $P$, Zhang B. The diagnostic value of serum miRNA-221-3p, miRNA-382-5p, and miRNA-4271 in ischemic stroke. J Stroke Cerebrovasc Dis 2017;26:1055-1060.

40. Zhou J, Chen L, Chen B, Huang S, Zeng C, Wu H, et al. Increased serum exosomal miR-134 expression in the acute ischemic stroke patients. BMC Neurol 2018;18:198.

41. Jickling GC, Ander BP, Shroff N, Orantia M, Stamova B, Dyk-

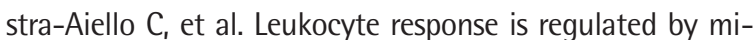
croRNA let7i in patients with acute ischemic stroke. Neurology 2016;87:2198-2205.

42. Yang $X$, Tang $X$, Sun $P$, Shi $Y$, Liu $K$, Hassan SH, et al. MicroRNA-15a/16-1 antagomir ameliorates ischemic brain injury in experimental stroke. Stroke 2017;48:1941-1947.

43. Ma M, Ma Y, Yi X, Guo R, Zhu W, Fan X, et al. Intranasal delivery of transforming growth factor-beta 1 in mice after stroke reduces infarct volume and increases neurogenesis in the subventricular zone. BMC Neurosci 2008;9:117.

44. Landgraf $P$, Rusu $M$, Sheridan $R$, Sewer $A$, lovino $N$, Aravin $A$, et al. A mammalian microRNA expression atlas based on small RNA library sequencing. Cell 2007;129:1401-1414.

45. Spinetti G, Fortunato O, Caporali A, Shantikumar S, Mar- chetti M, Meloni M, et al. MicroRNA-15a and microRNA-16 impair human circulating proangiogenic cell functions and are increased in the proangiogenic cells and serum of patients with critical limb ischemia. Circ Res 2013;112:335-346.

46. Maiese K. Cutting through the complexities of mTOR for the treatment of stroke. Curr Neurovasc Res 2014;11:177186.

47. Pomytkin I, Krasil'nikova I, Bakaeva Z, Surin A, Pinelis V. Excitotoxic glutamate causes neuronal insulin resistance by inhibiting insulin receptor/Akt/mTOR pathway. Mol Brain 2019;12:112.

48. van Solingen C, Seghers L, Bijkerk R, Duijs JM, Roeten MK, van Oeveren-Rietdijk AM, et al. Antagomir-mediated silencing of endothelial cell specific microRNA-126 impairs ischemia-induced angiogenesis. J Cell Mol Med 2009;13: 1577-1585.

49. Kim KJ, Cho CS, Kim WU. Role of placenta growth factor in cancer and inflammation. Exp Mol Med 2012;44:10-19.

50. Jakob $P$, Doerries $C$, Briand $S$, Mocharla P, Kränkel N, Besler

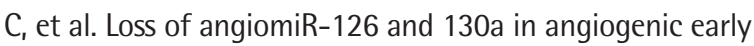
outgrowth cells from patients with chronic heart failure: role for impaired in vivo neovascularization and cardiac repair capacity. Circulation 2012;126:2962-2975.

51. Tang ST, Wang F, Shao M, Wang Y, Zhu HO. MicroRNA-126 suppresses inflammation in endothelial cells under hyperglycemic condition by targeting HMGB1. Vascul Pharmacol 2017;88:48-55.

52. Qu WS, Tian DS, Guo ZB, Fang J, Zhang Q, Yu ZY, et al. Inhibition of EGFR/MAPK signaling reduces microglial inflammatory response and the associated secondary damage in rats after spinal cord injury. J Neuroinflammation 2012;9:178.

53. Si W, Ye S, Ren Z, Liu X, Wu Z, Li Y, et al. miR-335 promotes stress granule formation to inhibit apoptosis by targeting ROCK2 in acute ischemic stroke. Int J Mol Med 2019;43: 1452-1466.

54. Gošev I, Zeljko M, Đurić Ž, Nikolić I, Gošev M, Ivčević S, et al. Epigenome alterations in aortic valve stenosis and its related left ventricular hypertrophy. Clin Epigenetics 2017;9:106.

55. Wang Y, Wang MD, Xia YP, Gao Y, Zhu YY, Chen SC, et al. MicroRNA-130a regulates cerebral ischemia-induced bloodbrain barrier permeability by targeting Homeobox A5. FASEB J 2018;32:935-944.

56. Huang RS, Gamazon ER, Ziliak D, Wen Y, Im HK, Zhang W, et al. Population differences in microRNA expression and biological implications. RNA Biol 2011;8:692-701.

57. Mompeón A, Ortega-Paz L, Vidal-Gómez X, Costa TJ, Pérez-Cremades D, Garcia-Blas $S$, et al. Disparate miRNA expression in serum and plasma of patients with acute myocar- 
dial infarction: a systematic and paired comparative analysis. Sci Rep 2020;10:5373.

58. Kroh EM, Parkin RK, Mitchell PS, Tewari M. Analysis of circulating microRNA biomarkers in plasma and serum using quantitative reverse transcription-PCR (qRT-PCR). Methods
2010;50:298-301.

59. Schwarzenbach $H$, da Silva AM, Calin G, Pantel K. Data normalization strategies for microRNA quantification. Clin Chem 2015;61:1333-1342. 
Supplementary Table 1. Study quality assessment using Newcastle-Ottawa scale for case-control studies

\begin{tabular}{|c|c|c|c|c|c|c|c|c|c|}
\hline \multirow[b]{2}{*}{ Study } & \multicolumn{4}{|c|}{ Selection } & \multirow{2}{*}{$\begin{array}{c}\text { Comparability } \\
\text { of cohorts }\end{array}$} & \multicolumn{3}{|c|}{ Outcome } & \multirow{2}{*}{$\begin{array}{l}\text { Total } \\
\text { score }\end{array}$} \\
\hline & $\begin{array}{l}\text { Adequate case } \\
\text { definition }\end{array}$ & $\begin{array}{c}\text { Representativeness } \\
\text { of cases }\end{array}$ & $\begin{array}{l}\text { Selection } \\
\text { of controls }\end{array}$ & $\begin{array}{l}\text { Definition } \\
\text { of controls }\end{array}$ & & $\begin{array}{c}\text { Ascertainment } \\
\text { of exposure }\end{array}$ & $\begin{array}{l}\text { Same method of } \\
\text { ascertainment }\end{array}$ & $\begin{array}{l}\text { Non-response } \\
\text { rate }\end{array}$ & \\
\hline $\begin{array}{l}\text { Leung et al. } \\
(2014)^{32}\end{array}$ & * & * & - & - & * & * & - & * & 5 \\
\hline $\begin{array}{l}\text { Tiedt et al. } \\
(2017)^{19}\end{array}$ & * & * & - & - & ** & * & - & * & 6 \\
\hline $\begin{array}{l}\text { Sepramaniam et al. } \\
(2014)^{20}\end{array}$ & * & * & - & - & * & * & - & - & 4 \\
\hline $\begin{array}{l}\text { Long et al. } \\
(2013)^{27}\end{array}$ & * & * & - & * & $* *$ & * & * & * & 8 \\
\hline $\begin{array}{l}\text { Huang et al. } \\
(2016)^{28}\end{array}$ & * & * & - & * & ** & * & - & * & 7 \\
\hline $\begin{array}{l}\text { Zhao et al. } \\
(2016)^{30}\end{array}$ & * & - & - & - & * & * & - & - & 3 \\
\hline $\begin{array}{l}\text { Li et al. } \\
(2018)^{22}\end{array}$ & * & - & - & * & $* *$ & * & - & * & 6 \\
\hline $\begin{array}{l}\text { Ma et al. } \\
(2019)^{23}\end{array}$ & * & - & - & * & ** & * & - & * & 6 \\
\hline $\begin{array}{l}\text { Yoo et al. } \\
(2019)^{24}\end{array}$ & * & - & - & - & $* *$ & * & - & * & 5 \\
\hline $\begin{array}{l}\text { Wang et al. } \\
(2016)^{36}\end{array}$ & * & - & - & - & - & * & - & - & 2 \\
\hline $\begin{array}{l}\text { Wang et al. } \\
(2014)^{33}\end{array}$ & - & * & * & * & * & * & - & * & 6 \\
\hline $\begin{array}{l}\text { Jickling et al. } \\
(2016)^{41}\end{array}$ & * & * & - & * & ** & * & - & * & 7 \\
\hline $\begin{array}{l}\text { Tian et al. } \\
(2016)^{25}\end{array}$ & - & * & - & - & ** & - & - & * & 4 \\
\hline $\begin{array}{l}\text { Chen et al. } \\
(2018)^{37}\end{array}$ & * & * & * & * & $* *$ & * & - & * & 8 \\
\hline $\begin{array}{l}\text { Jin et al. } \\
(2017)^{34}\end{array}$ & * & * & - & * & $* *$ & * & - & * & 7 \\
\hline $\begin{array}{l}\text { Xiang et al. } \\
(2017)^{21}\end{array}$ & * & - & - & - & * & * & - & * & 4 \\
\hline $\begin{array}{l}\text { Jin et al. } \\
(2018)^{35}\end{array}$ & * & * & - & * & $* *$ & * & - & * & 7 \\
\hline $\begin{array}{l}\text { Jia et al. } \\
(2015)^{38}\end{array}$ & * & * & * & * & $* *$ & * & - & * & 8 \\
\hline $\begin{array}{l}\text { Li et al. } \\
(2015)^{29}\end{array}$ & * & - & - & - & * & * & - & * & 4 \\
\hline $\begin{array}{l}\text { Liu et al. } \\
(2015)^{26}\end{array}$ & * & * & - & - & - & * & - & * & 3 \\
\hline $\begin{array}{l}\text { Wang et al. } \\
(2017)^{39}\end{array}$ & * & - & - & * & ** & * & - & * & 6 \\
\hline $\begin{array}{l}\text { Zhou et al. } \\
(2018)^{40}\end{array}$ & * & * & * & * & $* *$ & * & - & * & 8 \\
\hline
\end{tabular}

A maximum of one star is awarded for each numbered item within the selection and outcome categories and a maximum of two stars are awarded for comparability. A final quality score ranges from 0-9. Studies are classified as having high quality if score is 7-9, medium quality if score is 4-6 and low quality if score is 0-3. 
Supplementary Table 2. Gene Ontology (G0) analyses 